\title{
Nonlocal Theory of High-Temperature Superconductivity
}

\author{
Boris V. Alexeev \\ MIREA-Russian State Technological University, Moscow, Russia \\ Email: Boris.Vlad.Alexeev@gmail.com
}

How to cite this paper: Alexeev, B.V. (2021) Nonlocal Theory of High-Temperature Superconductivity. Journal of Modern Physics, 12, 552-593.

https://doi.org/10.4236/jmp.2021.125037

Received: March 10, 2021

Accepted: April 10, 2021

Published: April 13, 2021

Copyright $\odot 2021$ by author(s) and Scientific Research Publishing Inc. This work is licensed under the Creative Commons Attribution International License (CC BY 4.0).

http://creativecommons.org/licenses/by/4.0/

\begin{abstract}
The Boltzmann local physical kinetics forecasts the destruction of SC regime because of the heat movement of particles. Then, the most fundamental distinction between a strange metal and a conventional metal is the absence of well-defined quasi-particles. Here, we show that the mentioned "quasi-particles" are solitons, which are formed as a result of self-organization of ionized matter. Shortcomings of the Boltzmann physical kinetics consist in the local description of the transport processes on the level of infinitely small physical volumes as elements of diagnostics. The non-local physics leads to the theory superconductivity including the high temperature diapason. The generalized non-local non-stationary London's formula is derived.
\end{abstract}

\section{Keywords}

High Temperature Superconductivity, Generalized London's Formula, Nonlocal Physics, Transport Processes in Superconductors, Longitudinal Electromagnetic Waves, Solitons in Superconductors

\section{Introduction: Shortcomings of Boltzmann Physical Kinetics}

In $1872 \mathrm{~L}$ Boltzmann published his famous kinetic equation for the one-particle distribution function (DF) $f(\mathbf{r}, \mathbf{v}, t)$ [1] [2]. He expressed the equation in the form

$$
\frac{D f}{D t}=J^{s t}(f)
$$

where $J^{s t}$ is the collision ("stoß") integral, and

$$
\frac{D}{D t}=\frac{\partial}{\partial t}+\mathbf{v} \cdot \frac{\partial}{\partial \mathbf{r}}+\mathbf{F} \cdot \frac{\partial}{\partial \mathbf{v}}
$$

is the substantial (particle) derivative, $\mathbf{v}$ and $\mathbf{r}$ being the velocity and ra- 
dius-vector of the particle, respectively. Transport processes in open dissipative systems are considered in physical kinetics. Therefore, the kinetic description is inevitably related to the system diagnostics. Such an element of diagnostics in the case of theoretical description in physical kinetics is the concept of the physically infinitely small volume ( PhSV ). The correlation between theoretical description and system diagnostics is well-known in physics. Suffice it to recall the part played by test charge in electrostatics or by test circuit in the physics of magnetic phenomena.

Let us consider the hydrodynamic description in more detail from this point of view. Assume that we have two neighboring physically infinitely small volumes $\mathbf{P h S V}_{1}$ and $\mathbf{P h S V}_{2}$ in a non-equilibrium system. The one-particle distribution function (DF) $f_{s m, 1}\left(\mathbf{r}_{1}, \mathbf{v}, t\right)$ corresponds to the volume $\mathbf{P h S V}$, and the function $f_{s m, 2}\left(\mathbf{r}_{2}, \mathbf{v}, t\right)$ to the volume $\mathbf{P h S V} \mathbf{V}_{\mathbf{2}}$. It is assumed in a first approximation that $f_{s m, 1}\left(\mathbf{r}_{1}, \mathbf{v}, t\right)$ does not vary within $\mathbf{P h S V}_{\mathbf{1}}$, same as $f_{s m, 2}\left(\mathbf{r}_{2}, \mathbf{v}, t\right)$ does not vary within the neighboring volume $\mathbf{P h S V}_{\mathbf{2}}$. This assumption of locality is implicitly contained in the Boltzmann equation (BE) [3]-[11]. However, the assumption is too crude; PhSV is an open thermodynamic system.

The Boltzmann equation (BE) fully ignores non-local effects and contains only the local collision integral $J^{B}$. But these nonlocal effects are irrelevant only in equilibrium systems, where the kinetic approach goes into methods of statistical mechanics. As a result, the difficulties of classical Boltzmann physical kinetics arise. Conclusion:

1) Kinetic theory should be non-local.

2) The effect is of the order of Knudsen number.

3) The effect is due to the reduced description and is not related to the specific division of the physical system by the PhSV grid.

4) Accurate derivation of the kinetic equation with respect to the one-particle DF should lead to corrections of the order of the Knudsen number before uncoupling the Bogolyubov chain.

5) This means that in the Boltzmann equation, the terms of the order of the Knudsen number are lost, significant for both large and small Knudsen numbers.

6) The Boltzmann equation does not even belong to the class of minimal models, being only a "plausible" equation.

7) Boltzmann equation in this sense is the wrong equation.

\section{Generalization of the Boltzmann Kinetic Equation. Nonlocal Physical Kinetics}

A rigorous approach to the derivation of the kinetic equation for the distribution function (DF) $f_{1}\left(K E_{f_{1}}\right)$ is based on the hierarchy of the BogolyubovBorn-Green-Kirkwood-Yvon (BBGKY) equations [12] [13] [14] [15] [16]. The structure of the $K E_{f}$ is generally as follows

$$
\frac{D f}{D t}=J^{B}+J^{n l},
$$


where $J^{n l}$ is the non-local integral term incorporating in particular the time delay effect. The generalized Boltzmann physical kinetics, in essence, involves a local approximation

$$
J^{n l}=\frac{D}{D t}\left(\tau \frac{D f}{D t}\right)
$$

for the second collision integral, here $\tau$ being proportional to the mean time between the particle collisions. All of the known methods [3]-[11] of deriving kinetic equation relative to one-particle DF lead to approximation (2.2), including the method of many scales, the method of correlation functions, and the iteration method. We can draw here an analogy with the Bhatnagar-Gross-Krook (BGK, [17]) approximation for $J^{B}$,

$$
J^{B}=\frac{f_{0}-f}{\tau},
$$

which popularity as a means to represent the Boltzmann collision integral is due to the huge simplifications it offers.

In other words, the local Boltzmann collision integral admits approximation via the BGK algebraic expression (2.3), but more complicated non-local integral can be expressed as differential form (2.2). The ratio of the second to the first term on the right-hand side of Equation (2.1) is given to an order of magnitude as $J^{n l} / J^{B} \approx O\left(\mathrm{Kn}^{2}\right)$ and at large Knudsen numbers (defining as ratio of mean free path of particles to the character hydrodynamic length) these terms become of the same order of magnitude. It would seem that at small Knudsen numbers answering to hydrodynamic description the contribution from the second term on the right-hand side of Equation (2.1) is negligible.

This is not the case, however. When one goes over to the hydrodynamic approximation (by multiplying the kinetic equation by collision invariants and then integrating over velocities), the Boltzmann integral part vanishes, and the second term on the right-hand side of Equation (2.1) gives a single-order contribution in the generalized Navier-Stokes description. Mathematically, we cannot neglect a term with a small parameter in front of the higher derivative. Physically, the appearing additional terms are due to viscosity and they correspond to the small-scale Kolmogorov turbulence [8].

The integral term $J^{n l}$ turns out to be important both at small and large Knudsen numbers in the theory of transport processes.

Thus, $\tau D f / D t$ is the distribution function fluctuation, and we find

$$
\frac{D f^{a}}{D t}=J^{B}(f),
$$

where

$$
f^{a}=f-\tau \frac{D f}{D t} .
$$

Writing Equation (2.4) without taking into account Equation (2.5) makes the $\mathrm{BE}$ non-closed. From viewpoint of the fluctuation theory, Boltzmann employed 
the simplest possible closure procedure $f^{a}=f$. Then, the additional GBE terms (as compared to the $\mathrm{BE}$ ) are significant for any $\mathrm{Kn}$, and the order of magnitude of the difference between the $\mathrm{BE}$ and GBE solutions is impossible to tell beforehand. For GBE the generalized H-theorem is proven [9].

Madelung's quantum hydrodynamics is equivalent to the Schrödinger equation (SE) and furnishes the description of the quantum particle evolution in the form of Euler equation and continuity equation. Madelung's interpretation of SE (connected with wave function $\psi=\alpha \exp (i \beta)$ ) leads to the probability density $\rho=\alpha^{2}$ and velocity $\mathbf{v}=\frac{\partial}{\partial \mathbf{r}}(\beta \hbar / m)$. Schrödinger-Madelung quantum hydrodynamics does not lead to the energy equation in principle; then the Schrödinger-Madelung quantum mechanics cannot be used for the description of the dissipative processes.

The dependent variable $p$ in the energy equation of the generalized quantum hydrodynamics can be titled as the rest quantum pressure or simply quantum pressure.

Generalized Boltzmann physical kinetics brings the strict approximation of non-local effects in space and time and after transfer to the local approximation leads to parameter $\tau$, which on the quantum level corresponds to the uncertainty principle "time-energy".

The appearance of the nonlocal $\tau$ parameter is consistent with the Heisenberg uncertainty relation. But in principle generalized nonlocal kinetic equations (and therefore GHE) needn't in using of the "time-energy" uncertainty relation for estimation of the value of the non-locality parameter $\tau$. Moreover, the "time-energy" uncertainty relation does not produce the exact relations and from position of non-local physics is only the simplest estimation of the non-local effects. Really, let us consider two neighboring physically infinitely small volumes $\mathbf{P h S V}_{1}$ and $\mathbf{P h S V}_{2}$ in a non-equilibrium system. Obviously the time $\tau$ should tends to diminish with increasing of the velocities $u$ of particles invading in the nearest neighboring physically infinitely small volume $\left(\mathrm{PhSV}_{1}\right.$ or $\mathbf{P h S V}_{2}$ ):

$$
\tau=H_{\tau} / u^{n}
$$

But the value $\tau$ cannot depend on the velocity direction and naturally to tie $\tau$ with the particle kinetic energy, then

$$
\tau=H_{\tau} / m u^{2},
$$

where $H_{\tau}$ is a coefficient of proportionality, which reflects the state of physical system. In the simplest case $H_{\tau}$ is equal to Plank constant $\hbar$ and relation (2.7) became compatible with the Heisenberg relation. The non-locality parameter $\tau$ plays the same role as the transport coefficients in local hydrodynamics. The different models can be introduced for the $\tau$ definition, but the corresponding results not much different like in local kinetic theory for different models of the particles interaction.

In the general case, the parameter $\tau$ is the non-locality parameter; in quan- 
tum hydrodynamics, its magnitude is correlated with the Heisenberg "time-energy" uncertainty relation [18] [19] and with the Ehrenfest adiabatic theorem [20].

Now we can turn our attention to the quantum hydrodynamic description of individual particles. The abstract of the classical Madelung's paper [21] contains only one phrase: "It is shown that the Schrödinger equation for one-electron problems can be transformed into the form of hydrodynamic equations". The following conclusion of principal significance can be done from the previous consideration:

Madelung's quantum hydrodynamics is equivalent to the Schrödinger equation (SE) and leads to the description of the quantum particle evolution in the form of Euler equation and continuity equation. Generalized Boltzmann physical kinetics leads to the strict approximation of non-local effects in space and time and in the local limit leads to parameter $\tau$, which on the quantum level corresponds to the uncertainty principle "time-energy". Generalized hydrodynamic equations (GHE) lead to SE as a deep particular case of the generalized Boltzmann physical kinetics and therefore of non-local hydrodynamics.

Finally of Item 2 we can state that introduction of control volume by the reduced description for ensemble of particles of finite diameters leads to fluctuations (proportional to Knudsen number) of velocity moments in the volume. This fact leads to the significant reconstruction of the theory of transport processes. The violation of Bell's inequalities [22] is found for local statistical theories, and the transition to the non-local description is inevitable.

\section{Basic Quantum Nonlocal Hydrodynamic Equations. Superconducting Soliton Motion in the Two Component Physical System}

In general case the strict consideration leads to the following system of the non-local quantum hydrodynamic equations written in the Generalized Hydrodynamic Form (GHE) for multi-component species (see also [4]-[11]):

Continuity equation for species $\alpha$ :

$$
\begin{aligned}
& \frac{\partial}{\partial t}\left\{\rho_{\alpha}-\tau_{\alpha}\left[\frac{\partial \rho_{\alpha}}{\partial t}+\frac{\partial}{\partial \mathbf{r}} \cdot\left(\rho_{\alpha} \mathbf{v}_{0}\right)\right]\right\}+\frac{\partial}{\partial \mathbf{r}} \cdot\left\{\rho_{\alpha} \mathbf{v}_{0}-\tau_{\alpha}\left[\frac{\partial}{\partial t}\left(\rho_{\alpha} \mathbf{v}_{0}\right)\right.\right. \\
& \left.\left.+\frac{\partial}{\partial \mathbf{r}} \cdot\left(\rho_{\alpha} \mathbf{v}_{0} \mathbf{v}_{0}\right)+\overrightarrow{\mathrm{I}} \cdot \frac{\partial p_{\alpha}}{\partial \mathbf{r}}-\rho_{\alpha} \mathbf{F}_{\alpha}^{(1)}-\frac{q_{\alpha}}{m_{\alpha}} \rho_{\alpha} \mathbf{v}_{0} \times \mathbf{B}\right]\right\}=R_{\alpha} .
\end{aligned}
$$

Continuity equation for mixture:

$$
\begin{aligned}
& \frac{\partial}{\partial t}\left\{\rho-\sum_{\alpha} \tau_{\alpha}\left[\frac{\partial \rho_{\alpha}}{\partial t}+\frac{\partial}{\partial \mathbf{r}} \cdot\left(\rho_{\alpha} \mathbf{v}_{0}\right)\right]\right\} \\
& +\frac{\partial}{\partial \mathbf{r}} \cdot\left\{\rho \mathbf{v}_{0}-\sum_{\alpha} \tau_{\alpha}\left[\frac{\partial}{\partial t}\left(\rho_{\alpha} \mathbf{v}_{0}\right)+\frac{\partial}{\partial \mathbf{r}} \cdot\left(\rho_{\alpha} \mathbf{v}_{0} \mathbf{v}_{0}\right)\right.\right. \\
& \left.\left.+\overrightarrow{\mathrm{I}} \cdot \frac{\partial p_{\alpha}}{\partial \mathbf{r}}-\rho_{\alpha} \mathbf{F}_{\alpha}^{(1)}-\frac{q_{\alpha}}{m_{\alpha}} \rho_{\alpha} \mathbf{v}_{0} \times \mathbf{B}\right]\right\}=0 .
\end{aligned}
$$


Momentum equation for species $\alpha$ :

$$
\begin{aligned}
& \frac{\partial}{\partial t}\left\{\rho_{\alpha} \mathbf{v}_{0}-\tau_{\alpha}\left[\frac{\partial}{\partial t}\left(\rho_{\alpha} \mathbf{v}_{0}\right)+\frac{\partial}{\partial \mathbf{r}} \cdot \rho_{\alpha} \mathbf{v}_{0} \mathbf{v}_{0}+\frac{\partial p_{\alpha}}{\partial \mathbf{r}}-\rho_{\alpha} \mathbf{F}_{\alpha}^{(1)}-\frac{q_{\alpha}}{m_{\alpha}} \rho_{\alpha} \mathbf{v}_{0} \times \mathbf{B}\right]\right\} \\
& -\mathbf{F}_{\alpha}^{(1)}\left[\rho_{\alpha}-\tau_{\alpha}\left(\frac{\partial \rho_{\alpha}}{\partial t}+\frac{\partial}{\partial \mathbf{r}}\left(\rho_{\alpha} \mathbf{v}_{0}\right)\right]-\frac{q_{\alpha}}{m_{\alpha}}\left\{\rho_{\alpha} \mathbf{v}_{0}-\tau_{\alpha}\left[\frac{\partial}{\partial t}\left(\rho_{\alpha} \mathbf{v}_{0}\right)\right.\right.\right. \\
& \left.\left.+\frac{\partial}{\partial \mathbf{r}} \cdot \rho_{\alpha} \mathbf{v}_{0} \mathbf{v}_{0}+\frac{\partial p_{\alpha}}{\partial \mathbf{r}}-\rho_{\alpha} \mathbf{F}_{\alpha}^{(1)}-\frac{q_{\alpha}}{m_{\alpha}} \rho_{\alpha} \mathbf{v}_{0} \times \mathbf{B}\right]\right\} \times \mathbf{B}+\frac{\partial}{\partial \mathbf{r}} \cdot\left\{\rho_{\alpha} \mathbf{v}_{0} \mathbf{v}_{0}+p_{\alpha} \overrightarrow{\mathrm{I}}\right. \\
& -\tau_{\alpha}\left[\frac{\partial}{\partial t}\left(\rho_{\alpha} \mathbf{v}_{0} \mathbf{v}_{0}+p_{\alpha} \overleftrightarrow{\mathrm{I}}\right)+\frac{\partial}{\partial \mathbf{r}} \cdot \rho_{\alpha}\left(\mathbf{v}_{0} \mathbf{v}_{0}\right) \mathbf{v}_{0}+2 \overleftrightarrow{\mathrm{I}}\left(\frac{\partial}{\partial \mathbf{r}} \cdot\left(p_{\alpha} \mathbf{v}_{0}\right)\right)+\frac{\partial}{\partial \mathbf{r}} \cdot\left(\overleftrightarrow{\mathrm{I}} p_{\alpha} \mathbf{v}_{0}\right)\right. \\
& \left.\left.-\mathbf{F}_{\alpha}^{(1)} \rho_{\alpha} \mathbf{v}_{0}-\rho_{\alpha} \mathbf{v}_{0} \mathbf{F}_{\alpha}^{(1)}-\frac{q_{\alpha}}{m_{\alpha}} \rho_{\alpha}\left[\mathbf{v}_{0} \times \mathbf{B}\right] \mathbf{v}_{0}-\frac{q_{\alpha}}{m_{\alpha}} \rho_{\alpha} \mathbf{v}_{0}\left[\mathbf{v}_{0} \times \mathbf{B}\right]\right]\right\} \\
& =\int m_{\alpha} \mathbf{v}_{\alpha} J_{\alpha}^{\text {st,el }} \mathrm{d} \mathbf{v}_{\alpha}+\int m_{\alpha} \mathbf{v}_{\alpha} J_{\alpha}^{\text {st,inel }} \mathrm{d} \mathbf{v}_{\alpha} .
\end{aligned}
$$

Momentum equation for mixture

$$
\begin{aligned}
& \frac{\partial}{\partial t}\left\{\rho \mathbf{v}_{0}-\sum_{\alpha} \tau_{\alpha}\left[\frac{\partial}{\partial t}\left(\rho_{\alpha} \mathbf{v}_{0}\right)+\frac{\partial}{\partial \mathbf{r}} \cdot \rho_{\alpha} \mathbf{v}_{0} \mathbf{v}_{0}+\frac{\partial p_{\alpha}}{\partial \mathbf{r}}-\rho_{\alpha} \mathbf{F}_{\alpha}^{(1)}-\frac{q_{\alpha}}{m_{\alpha}} \rho_{\alpha} \mathbf{v}_{0} \times \mathbf{B}\right]\right\} \\
& -\sum_{\alpha} \mathbf{F}_{\alpha}^{(1)}\left[\rho_{\alpha}-\tau_{\alpha}\left(\frac{\partial \rho_{\alpha}}{\partial t}+\frac{\partial}{\partial \mathbf{r}}\left(\rho_{\alpha} \mathbf{v}_{0}\right)\right]\right]-\sum_{\alpha} \frac{q_{\alpha}}{m_{\alpha}}\left\{\rho_{\alpha} \mathbf{v}_{0}-\tau_{\alpha}\left[\frac{\partial}{\partial t}\left(\rho_{\alpha} \mathbf{v}_{0}\right)\right.\right. \\
& \left.\left.+\frac{\partial}{\partial \mathbf{r}} \cdot \rho_{\alpha} \mathbf{v}_{0} \mathbf{v}_{0}+\frac{\partial p_{\alpha}}{\partial \mathbf{r}}-\rho_{\alpha} \mathbf{F}_{\alpha}^{(1)}-\frac{q_{\alpha}}{m_{\alpha}} \rho_{\alpha} \mathbf{v}_{0} \times \mathbf{B}\right]\right\} \times \mathbf{B} \\
& +\frac{\partial}{\partial \mathbf{r}} \cdot\left\{\rho \mathbf{v}_{0} \mathbf{v}_{0}+p \overrightarrow{\mathrm{I}}-\sum_{\alpha} \tau_{\alpha}\left[\frac{\partial}{\partial t}\left(\rho_{\alpha} \mathbf{v}_{0} \mathbf{v}_{0}+p_{\alpha} \overrightarrow{\mathrm{I}}\right)+\frac{\partial}{\partial \mathbf{r}} \cdot \rho_{\alpha}\left(\mathbf{v}_{0} \mathbf{v}_{0}\right) \mathbf{v}_{0}\right.\right. \\
& +2 \overrightarrow{\mathrm{I}}\left(\frac{\partial}{\partial \mathbf{r}} \cdot\left(p_{\alpha} \mathbf{v}_{0}\right)\right)+\frac{\partial}{\partial \mathbf{r}} \cdot\left(\overleftrightarrow{\mathrm{I}} p_{\alpha} \mathbf{v}_{0}\right)-\mathbf{F}_{\alpha}^{(1)} \rho_{\alpha} \mathbf{v}_{0}-\rho_{\alpha} \mathbf{v}_{0} \mathbf{F}_{\alpha}^{(1)} \\
& \left.\left.-\frac{q_{\alpha}}{m_{\alpha}} \rho_{\alpha}\left[\mathbf{v}_{0} \times \mathbf{B}\right] \mathbf{v}_{0}-\frac{q_{\alpha}}{m_{\alpha}} \rho_{\alpha} \mathbf{v}_{0}\left[\mathbf{v}_{0} \times \mathbf{B}\right]\right]\right\}=0
\end{aligned}
$$

Energy equation for $\alpha$ species

$$
\begin{aligned}
& \frac{\partial}{\partial t}\left\{\frac{\rho_{\alpha} v_{0}^{2}}{2}+\frac{3}{2} p_{\alpha}+\varepsilon_{\alpha} n_{\alpha}-\tau_{\alpha}\left[\frac{\partial}{\partial t}\left(\frac{\rho_{\alpha} v_{0}^{2}}{2}+\frac{3}{2} p_{\alpha}+\varepsilon_{\alpha} n_{\alpha}\right)\right.\right. \\
& \left.\left.+\frac{\partial}{\partial \mathbf{r}} \cdot\left(\frac{1}{2} \rho_{\alpha} v_{0}^{2} \mathbf{v}_{0}+\frac{5}{2} p_{\alpha} \mathbf{v}_{0}+\varepsilon_{\alpha} n_{\alpha} \mathbf{v}_{0}\right)-\mathbf{F}_{\alpha}^{(1)} \cdot \rho_{\alpha} \mathbf{v}_{0}\right]\right\} \\
& +\frac{\partial}{\partial \mathbf{r}} \cdot\left\{\frac{1}{2} \rho_{\alpha} v_{0}^{2} \mathbf{v}_{0}+\frac{5}{2} p_{\alpha} \mathbf{v}_{0}+\varepsilon_{\alpha} n_{\alpha} \mathbf{v}_{0}-\tau_{\alpha}\left[\frac { \partial } { \partial t } \left(\frac{1}{2} \rho_{\alpha} v_{0}^{2} \mathbf{v}_{0}\right.\right.\right. \\
& \left.+\frac{5}{2} p_{\alpha} \mathbf{v}_{0}+\varepsilon_{\alpha} n_{\alpha} \mathbf{v}_{0}\right)+\frac{\partial}{\partial \mathbf{r}} \cdot\left(\frac{1}{2} \rho_{\alpha} v_{0}^{2} \mathbf{v}_{0} \mathbf{v}_{0}+\frac{7}{2} p_{\alpha} \mathbf{v}_{0} \mathbf{v}_{0}+\frac{1}{2} p_{\alpha} v_{0}^{2} \overrightarrow{\mathrm{I}}\right. \\
& \left.+\frac{5}{2} \frac{p_{\alpha}^{2}}{\rho_{\alpha}} \overleftrightarrow{\mathrm{I}}^{2} \varepsilon_{\alpha} n_{\alpha} \mathbf{v}_{0} \mathbf{v}_{0}+\varepsilon_{\alpha} \frac{p_{\alpha}}{m_{\alpha}} \overleftrightarrow{\mathrm{I}}\right)-\rho_{\alpha} \mathbf{F}_{\alpha}^{(1)} \cdot \mathbf{v}_{0} \mathbf{v}_{0}-p_{\alpha} \mathbf{F}_{\alpha}^{(1)} \cdot \overrightarrow{\mathrm{I}} \\
& -\frac{1}{2} \rho_{\alpha} v_{0}^{2} \mathbf{F}_{\alpha}^{(1)}-\frac{3}{2} \mathbf{F}_{\alpha}^{(1)} p_{\alpha}-\frac{\rho_{\alpha} v_{0}^{2}}{2} \frac{q_{\alpha}}{m_{\alpha}}\left[\mathbf{v}_{0} \times \mathbf{B}\right]-\frac{5}{2} p_{\alpha} \frac{q_{\alpha}}{m_{\alpha}}\left[\mathbf{v}_{0} \times \mathbf{B}\right]
\end{aligned}
$$




$$
\begin{aligned}
& \left.\left.-\varepsilon_{\alpha} n_{\alpha} \frac{q_{\alpha}}{m_{\alpha}}\left[\mathbf{v}_{0} \times \mathbf{B}\right]-\varepsilon_{\alpha} n_{\alpha} \mathbf{F}_{\alpha}^{(1)}\right]\right\}-\left\{\mathbf{v}_{0} \cdot \sum_{\alpha} \rho_{\alpha} \mathbf{F}_{\alpha}^{(1)}\right. \\
& \left.\left.\left.+\frac{\partial}{\partial \mathbf{r}} \cdot \rho_{\alpha} \mathbf{v}_{0} \mathbf{v}_{0}+\frac{\partial}{\partial \mathbf{r}} \cdot p_{\alpha} \overrightarrow{\mathrm{I}}-\rho_{\alpha} \mathbf{F}_{\alpha}^{(1)}-q_{\alpha} n_{\alpha}\left[\mathbf{v}_{0} \times \mathbf{B}\right]\right)\right]\right\} \\
& =\int\left(\frac{m_{\alpha} v_{\alpha}^{2}}{2}+\varepsilon_{\alpha}\right) J_{\alpha}^{s t, e l} \mathrm{~d} \mathbf{v}_{\alpha}+\int\left(\frac{m_{\alpha} v_{\alpha}^{2}}{2}+\varepsilon_{\alpha}\right) J_{\alpha}^{s t, \text { inel }} \mathrm{d} \mathbf{v}_{\alpha} .
\end{aligned}
$$

Energy equation for mixture:

$$
\begin{aligned}
& \frac{\partial}{\partial t}\left\{\frac{\rho v_{0}^{2}}{2}+\frac{3}{2} p+\sum_{\alpha} \varepsilon_{\alpha} n_{\alpha}-\sum_{\alpha} \tau_{\alpha}\left[\frac{\partial}{\partial t}\left(\frac{\rho_{\alpha} v_{0}^{2}}{2}+\frac{3}{2} p_{\alpha}+\varepsilon_{\alpha} n_{\alpha}\right)\right.\right. \\
& \left.\left.+\frac{\partial}{\partial \mathbf{r}} \cdot\left(\frac{1}{2} \rho_{\alpha} v_{0}^{2} \mathbf{v}_{0}+\frac{5}{2} p_{\alpha} \mathbf{v}_{0}+\varepsilon_{\alpha} n_{\alpha} \mathbf{v}_{0}\right)-\mathbf{F}_{\alpha}^{(1)} \cdot \rho_{\alpha} \mathbf{v}_{0}\right]\right\} \\
& +\frac{\partial}{\partial \mathbf{r}} \cdot\left\{\frac{1}{2} \rho v_{0}^{2} \mathbf{v}_{0}+\frac{5}{2} p \mathbf{v}_{0}+\mathbf{v}_{0} \sum_{\alpha} \varepsilon_{\alpha} n_{\alpha}-\sum_{\alpha} \tau_{\alpha}\left[\frac { \partial } { \partial t } \left(\frac{1}{2} \rho_{\alpha} v_{0}^{2} \mathbf{v}_{0}\right.\right.\right. \\
& \left.+\frac{5}{2} p_{\alpha} \mathbf{v}_{0}+\varepsilon_{\alpha} n_{\alpha} \mathbf{v}_{0}\right)+\frac{\partial}{\partial \mathbf{r}} \cdot\left(\frac{1}{2} \rho_{\alpha} v_{0}^{2} \mathbf{v}_{0} \mathbf{v}_{0}+\frac{7}{2} p_{\alpha} \mathbf{v}_{0} \mathbf{v}_{0}+\frac{1}{2} p_{\alpha} v_{0}^{2} \overrightarrow{\mathrm{I}}\right. \\
& \left.+\frac{5}{2} \frac{p_{\alpha}^{2}}{\rho_{\alpha}} \overrightarrow{\mathrm{I}}+\varepsilon_{\alpha} n_{\alpha} \mathbf{v}_{0} \mathbf{v}_{0}+\varepsilon_{\alpha} \frac{p_{\alpha}}{m_{\alpha}} \overrightarrow{\mathrm{I}}\right)-\rho_{\alpha} \mathbf{F}_{\alpha}^{(1)} \cdot \mathbf{v}_{0} \mathbf{v}_{0}-p_{\alpha} \mathbf{F}_{\alpha}^{(1)} \cdot \overrightarrow{\mathrm{I}} \\
& -\frac{1}{2} \rho_{\alpha} v_{0}^{2} \mathbf{F}_{\alpha}^{(1)}-\frac{3}{2} \mathbf{F}_{\alpha}^{(1)} p_{\alpha}-\frac{\rho_{\alpha} v_{0}^{2}}{2} \frac{q_{\alpha}}{m_{\alpha}}\left[\mathbf{v}_{0} \times \mathbf{B}\right]-\frac{5}{2} p_{\alpha} \frac{q_{\alpha}}{m_{\alpha}}\left[\mathbf{v}_{0} \times \mathbf{B}\right] \\
& \left.\left.-\varepsilon_{\alpha} n_{\alpha} \frac{q_{\alpha}}{m_{\alpha}}\left[\mathbf{v}_{0} \times \mathbf{B}\right]-\varepsilon_{\alpha} n_{\alpha} \mathbf{F}_{\alpha}^{(1)}\right]\right\}-\left\{\mathbf{v}_{0} \cdot \sum_{\alpha} \rho_{\alpha} \mathbf{F}_{\alpha}^{(1)}\right. \\
& -\sum_{\alpha} \tau_{\alpha}\left[\mathbf { F } _ { \alpha } ^ { ( 1 ) } \cdot \left(\frac{\partial}{\partial t}\left(\rho_{\alpha} \mathbf{v}_{0}\right)+\frac{\partial}{\partial \mathbf{r}} \cdot \rho_{\alpha} \mathbf{v}_{0} \mathbf{v}_{0}\right.\right. \\
& \left.\left.\left.+\frac{\partial}{\partial \mathbf{r}} \cdot p_{\alpha} \overrightarrow{\mathrm{I}}-\rho_{\alpha} \mathbf{F}_{\alpha}^{(1)}-q_{\alpha} n_{\alpha}\left[\mathbf{v}_{0} \times \mathbf{B}\right]\right)\right]\right\}=0 .
\end{aligned}
$$

Here $\mathbf{F}_{\alpha}^{(1)}$ are the forces (acting on the mass unit) of the non-magnetic origin, B -magnetic induction, $\overrightarrow{\mathrm{I}}$ - unit tensor, $q_{\alpha}$-charge of the $\alpha$ -component particle, $p_{\alpha}$-static pressure for $\alpha$-component, $\varepsilon_{\alpha}$-internal energy for the particles of $\alpha$-component, $\mathbf{v}_{0}$-hydrodynamic velocity for mixture, $\tau_{\alpha}$-non-local parameter.

In the following we intend to obtain the soliton's type of solution of the generalized hydrodynamic equations (GHE). The non-stationary $1 \mathrm{D}$ model will be used with taking into account the energy equation, external forces and non-locality parameter $\tau$ defined by the "time-energy" uncertainty relation of Heisenberg. Then GHE contain Poisson equation (reflected fluctuations of charges and flux of the charges density), two continuity equations for positive (lattice ions) and negative (electrons) species, momentum equation and two energy equations for positive and negative species. This system of six non-stationary $1 \mathrm{D}$ equations is written as:

(Generalized Poisson equation): 


$$
\frac{\partial^{2} \psi}{\partial x^{2}}=-4 \pi e\left\{\left[n_{i}-\tau_{i}\left(\frac{\partial n_{i}}{\partial t}+\frac{\partial}{\partial x}\left(n_{i} u\right)\right)\right]-\left[n_{e}-\tau_{e}\left(\frac{\partial n_{e}}{\partial t}+\frac{\partial}{\partial x}\left(n_{e} u\right)\right)\right]\right\} .
$$

(Continuity equation for ions):

$$
\begin{aligned}
& \frac{\partial}{\partial t}\left\{\rho_{i}-\tau_{i}\left[\frac{\partial \rho_{i}}{\partial t}+\frac{\partial}{\partial x}\left(\rho_{i} u\right)\right]\right\} \\
& +\frac{\partial}{\partial x}\left\{\rho_{i} u-\tau_{i}\left[\frac{\partial}{\partial t}\left(\rho_{i} u\right)+\frac{\partial}{\partial x}\left(\rho_{i} u^{2}\right)+\frac{\partial p_{i}}{\partial x}-\rho_{i} F_{i}\right]\right\}=0
\end{aligned} .
$$

(Continuity equation for electrons):

$$
\begin{aligned}
& \frac{\partial}{\partial t}\left\{\rho_{e}-\tau_{e}\left[\frac{\partial \rho_{e}}{\partial t}+\frac{\partial}{\partial x}\left(\rho_{e} u\right)\right]\right\} \\
& +\frac{\partial}{\partial x}\left\{\rho_{e} u-\tau_{e}\left[\frac{\partial}{\partial t}\left(\rho_{e} u\right)+\frac{\partial}{\partial x}\left(\rho_{e} u^{2}\right)+\frac{\partial p_{e}}{\partial x}-\rho_{e} F_{e}\right]\right\}=0
\end{aligned} .
$$

(Momentum equation):

$$
\begin{aligned}
& \frac{\partial}{\partial t}\left\{\rho u-\tau_{i}\left[\frac{\partial}{\partial t}\left(\rho_{i} u\right)+\frac{\partial}{\partial x}\left(p_{i}+\rho_{i} u^{2}\right)-\rho_{i} F_{i}\right]\right. \\
& \left.-\tau_{e}\left[\frac{\partial}{\partial t}\left(\rho_{e} u\right)+\frac{\partial}{\partial x}\left(p_{e}+\rho_{e} u^{2}\right)-\rho_{e} F_{e}\right]\right\} \\
& -\rho_{i} F_{i}-\rho_{e} F_{e}+F_{i} \tau_{i}\left(\frac{\partial \rho_{i}}{\partial t}+\frac{\partial}{\partial x}\left(\rho_{i} u\right)\right)+F_{e} \tau_{e}\left(\frac{\partial \rho_{e}}{\partial t}+\frac{\partial}{\partial x}\left(\rho_{e} u\right)\right) \\
& +\frac{\partial}{\partial x}\left\{\rho u^{2}+p-\tau_{i}\left[\frac{\partial}{\partial t}\left(\rho_{i} u^{2}+p_{i}\right)+\frac{\partial}{\partial x}\left(\rho_{i} u^{3}+3 p_{i} u\right)-2 \rho_{i} u F_{i}\right]\right. \\
& \left.-\tau_{e}\left[\frac{\partial}{\partial t}\left(\rho_{e} u^{2}+p_{e}\right)+\frac{\partial}{\partial x}\left(\rho_{e} u^{3}+3 p_{e} u\right)\right]-2 \rho_{e} u F_{e}\right\}=0 .
\end{aligned}
$$

(Energy equation for ions):

$$
\begin{aligned}
& \frac{\partial}{\partial t}\left\{\rho_{i} u^{2}+3 p_{i}-\tau_{i}\left[\frac{\partial}{\partial t}\left(\rho_{i} u^{2}+3 p_{i}\right)+\frac{\partial}{\partial x}\left(\rho_{i} u^{3}+5 p_{i} u\right)-2 \rho_{i} F_{i} u\right]\right\} \\
& +\frac{\partial}{\partial x}\left\{\rho_{i} u^{3}+5 p_{i} u-\tau_{i}\left[\frac{\partial}{\partial t}\left(\rho_{i} u^{3}+5 p_{i} u\right)\right.\right. \\
& \left.\left.+\frac{\partial}{\partial x}\left(\rho_{i} u^{4}+8 p_{i} u^{2}+5 \frac{p_{i}^{2}}{\rho_{i}}\right)-F_{i}\left(3 \rho_{i} u^{2}+5 p_{i}\right)\right]\right\} \\
& -2 u \rho_{i} F_{i}+2 \tau_{i} F_{i}\left[\frac{\partial}{\partial t}\left(\rho_{i} u\right)+\frac{\partial}{\partial x}\left(\rho_{i} u^{2}+p_{i}\right)-\rho_{i} F_{i}\right]=-\frac{p_{i}-p_{e}}{\tau_{e i}}
\end{aligned}
$$

(Energy equation for electrons):

$$
\begin{aligned}
& \frac{\partial}{\partial t}\left\{\rho_{e} u^{2}+3 p_{e}-\tau_{e}\left[\frac{\partial}{\partial t}\left(\rho_{e} u^{2}+3 p_{e}\right)+\frac{\partial}{\partial x}\left(\rho_{e} u^{3}+5 p_{e} u\right)-2 \rho_{e} F_{e} u\right]\right\} \\
& +\frac{\partial}{\partial x}\left\{\rho_{e} u^{3}+5 p_{e} u-\tau_{e}\left[\frac{\partial}{\partial t}\left(\rho_{e} u^{3}+5 p_{e} u\right)\right.\right. \\
& \left.\left.+\frac{\partial}{\partial x}\left(\rho_{e} u^{4}+8 p_{e} u^{2}+5 \frac{p_{e}^{2}}{\rho_{e}}\right)-F_{e}\left(3 \rho_{e} u^{2}+5 p_{e}\right)\right]\right\}
\end{aligned}
$$




$$
-2 u \rho_{e} F_{e}+2 \tau_{e} F_{e}\left[\frac{\partial}{\partial t}\left(\rho_{e} u\right)+\frac{\partial}{\partial x}\left(\rho_{e} u^{2}+p_{e}\right)-\rho_{e} F_{e}\right]=-\frac{p_{e}-p_{i}}{\tau_{e i}},
$$

where $u$ is velocity of the directed motion of combined quantum object (phonon-electron), $n_{i}$ and $n_{e}$-numerical density of the charged species, $F_{i}$ and $F_{e}$-forces (of potential and non-potential origin), acting on the mass unit of the charged particles. The right hand sides of the energy equations are written in the relaxation forms following from BGK kinetic approximation.

For acting potential forces of the electrical origin the relations are valid

$$
\begin{aligned}
& F_{i}^{(p o t)}=-\frac{e}{m_{i}} \frac{\partial \psi}{\partial x}, \\
& F_{e}^{(p o t)}=\frac{e}{m_{e}} \frac{\partial \psi}{\partial x},
\end{aligned}
$$

where $\psi$-scalar potential.

Let us introduce approximations for $\tau_{i}$ and $\tau_{i}$ using (2.7)

$$
\tau_{i}=\frac{\hbar}{m_{i} u^{2}}, \quad \tau_{e}=\frac{\hbar}{m_{e} u^{2}} .
$$

For electron-phonon non-local parameter $\tau_{e i}$ the following relation is applicable

$$
\frac{1}{\tau_{e i}}=\frac{1}{\tau_{e}}+\frac{1}{\tau_{i}}
$$

or

$$
\frac{1}{\tau_{e i}}=\frac{\tau_{e}+\tau_{i}}{\tau_{e} \tau_{i}}=\frac{\frac{\hbar}{m_{e} u^{2}}+\frac{\hbar}{m_{i} u^{2}}}{\frac{\hbar^{2}}{u^{4}} \frac{1}{m_{e} m_{i}}}=\frac{u^{2}}{\hbar}\left(m_{e}+m_{i}\right) .
$$

Formula (3.17) is obvious consequence of uncertainty relation for combined particle which mass is $m_{i}+m_{e}$. Energy equation of the generalized quantum hydrodynamics contains pressures $p_{i}, p_{e}$, which can be named as the quantum pressure of the non-local origin. In the definite sense these pressures can be considered as analog of the Bose condensate pressure.

\section{Combined Quantum Solitons in the Self-Consistent Electric Field}

Let us formulate the problem in detail. The non-stationary $1 \mathrm{D}$ motion of the combined phonon-electron soliton is considered under influence of the selfconsistent electric forces of the potential and non-potential origin. It should be shown that mentioned soliton can exists without a chemical bond formation. We introduce the coordinate system moving along the positive direction of the $x$ axis in $1 \mathrm{D}$ space with the velocity $C=u_{0}$, which $C$ is equal to the phase velocity of this quantum object.

$$
\xi=x-C t \text {. }
$$


Taking into account de Broglie relation we should wait that the group velocity $u_{g}$ is equal to $2 u_{0}$. Really the energy of a relativistic particle is

$$
E=m c^{2}
$$

where

$$
m=m_{0}\left(1-\frac{v_{g}^{2}}{c^{2}}\right)^{-1 / 2}
$$

and $c$ is the light velocity, $v_{g}$ is the group velocity, $m_{0}$-the particle rest mass. Relation (4.2) can be written as

$$
E=\mathrm{p} \frac{c^{2}}{v_{g}}
$$

where

$$
\mathrm{p}=m v_{g}
$$

is the particle impulse. In the non-relativistic approach the relation (4.4) takes the form

$$
E=\frac{1}{2} m_{0} v_{g}^{2} .
$$

Using the dualism principle in the de Broglie interpretation we have for the particle energy

$$
E=\hbar \omega=\hbar k v_{p h}
$$

where $\omega$ is the circular frequency, $v_{p h}=\frac{\omega}{\kappa}$-the phase velocity, $\kappa=2 \pi / \lambda$ is the wave number and $\lambda$ is the wave length. Correspondingly the particle impulse $\mathrm{p}$ is

$$
\mathrm{p}=\hbar k
$$

and using (4.8),

$$
E=\mathrm{p} v_{p h} .
$$

Then in the non-relativistic case we have

$$
E=\frac{1}{2} m_{0} v_{g}^{2}=\frac{1}{2} \mathrm{p} v_{g} .
$$

From (4.9) and (4.10) for the non-relativistic case one obtains

$$
v_{g}=2 v_{p h} .
$$

Then we should wait that the indestructible soliton has the velocity $v_{p h}$ in the coordinate system moving with the phase velocity $v_{p h}$. If we pass on the moving coordinate system, all dependent hydrodynamic values will be functions of $(\xi, t)$. But we investigate the possibility of the creation of the combined quantum object of the soliton type. For this case the explicit time dependence of solutions does not exist in mentioned coordinate system moving with the phase velocity $u_{0}$. 
Write down the system of Equations (3.7)-(3.12) for the two component mixture of charged particles (without taking into account the component's internal energy) in the dimensionless form, where dimensionless symbols are marked by tildes. We introduce the scales for velocity $[u]=u_{0}$, for coordinate $x \frac{\hbar}{m_{e} u_{0}}=x_{0}$, for the potential scale $\psi_{0}=\frac{m_{e}}{e} u_{0}^{2}$ and for the density scale $\rho_{0}=\frac{m_{e}^{4}}{4 \pi \hbar^{2} e^{2}} u_{0}^{4} \quad(e$ is absolute electron charge).

Generalized Poisson Equation (3.7) takes the form

$$
\frac{\partial^{2} \tilde{\psi}}{\partial \tilde{\xi}^{2}}=-\left\{\frac{m_{e}}{m_{i}}\left[\tilde{\rho}_{i}-\frac{1}{u^{2}} \frac{m_{e}}{m_{i}}\left(-\frac{\partial \tilde{\rho}_{i}}{\partial \tilde{\xi}}+\frac{\partial}{\partial \tilde{\xi}}\left(\tilde{\rho}_{i} \tilde{u}\right)\right)\right]-\left[\tilde{\rho}_{e}-\frac{1}{\tilde{u}^{2}}\left(-\frac{\partial \tilde{\rho}_{e}}{\partial \tilde{\xi}}+\frac{\partial}{\partial \tilde{\xi}}\left(\tilde{\rho}_{e} \tilde{u}\right)\right)\right]\right\} .
$$

Scaling forces are

$$
\begin{gathered}
\rho_{i} F_{i}=-\frac{u_{0}^{2}}{x_{0}} \rho_{0} \frac{m_{e}}{m_{i}} \frac{\partial \tilde{\psi}}{\partial \tilde{\xi}} \tilde{\rho}_{i}, \\
\rho_{e} F_{e}=\frac{u_{0}^{2}}{x_{0}} \rho_{0} \frac{\partial \tilde{\psi}}{\partial \tilde{\xi}} \tilde{\rho}_{e} .
\end{gathered}
$$

Analogical transformations should be applied to other equations of the system (3.7)-(3.12). As result one obtains the six non-linear dimensionless ordinary differential equations

$$
\begin{aligned}
\frac{\partial^{2} \tilde{\psi}}{\partial \tilde{\xi}^{2}}= & -\left\{\frac{m_{e}}{m_{i}}\left[\tilde{\rho}_{i}-\frac{1}{\tilde{u}^{2}} \frac{m_{e}}{m_{i}}\left(-\frac{\partial \tilde{\rho}_{i}}{\partial \tilde{\xi}}+\frac{\partial}{\partial \tilde{\xi}}\left(\tilde{\rho}_{i} \tilde{u}\right)\right]-\left[\tilde{\rho}_{e}-\frac{1}{\tilde{u}^{2}}\left(-\frac{\partial \tilde{\rho}_{e}}{\partial \tilde{\xi}}+\frac{\partial}{\partial \tilde{\xi}}\left(\tilde{\rho}_{e} \tilde{u}\right)\right)\right]\right\},\right. \\
\frac{\partial \tilde{\rho}_{i}}{\partial \tilde{\xi}}- & \frac{\partial \tilde{\rho}_{i} \tilde{u}}{\partial \tilde{\xi}}+\frac{m_{e}}{m_{i}} \frac{\partial}{\partial \tilde{\xi}}\left\{\frac{1}{\tilde{u}^{2}}\left[\frac{\partial}{\partial \tilde{\xi}}\left(\tilde{p}_{i}+\tilde{\rho}_{i}+\tilde{\rho}_{i} \tilde{u}^{2}-2 \tilde{\rho}_{i} \tilde{u}_{i}\right)+\frac{m_{e}}{m_{i}} \tilde{\rho}_{i} \frac{\partial \tilde{\psi}}{\partial \tilde{\xi}}\right]\right\}=0,(4.16) \\
& \frac{\partial \tilde{\rho}_{e}}{\partial \tilde{\xi}}-\frac{\partial \tilde{\rho}_{e} \tilde{u}}{\partial \tilde{\xi}}+\frac{\partial}{\partial \tilde{\xi}}\left\{\frac{1}{\tilde{u}^{2}}\left[\frac{\partial}{\partial \tilde{\xi}}\left(\tilde{p}_{e}+\tilde{\rho}_{e}+\tilde{\rho}_{e} \tilde{u}^{2}-2 \tilde{\rho}_{e} \tilde{u}_{e}\right)-\tilde{\rho}_{e} \frac{\partial \tilde{\psi}}{\partial \tilde{\xi}}\right]\right\}=0,(4.17) \\
& \frac{\partial}{\partial \tilde{\xi}}\left\{\left(\tilde{\rho}_{i}+\tilde{\rho}_{e}\right) \tilde{u}^{2}+\tilde{p}_{i}+\tilde{p}_{e}-\left(\tilde{\rho}_{i}+\tilde{\rho}_{e}\right) \tilde{u}\right\} \\
& +\frac{\partial}{\partial \tilde{\xi}}\left\{\frac{1}{\tilde{u}^{2}} \frac{m_{e}}{m_{i}}\left[\frac{\partial}{\partial \tilde{\xi}}\left(2 \tilde{p}_{i}+2 \tilde{p}_{i} \tilde{u}^{2}-\tilde{\rho}_{i} \tilde{u}-\tilde{\rho}_{i} \tilde{u}^{3}-3 \tilde{p}_{i} \tilde{u}\right)+\tilde{\rho}_{i} \frac{m_{e}}{m_{i}} \frac{\partial \tilde{\psi}}{\partial \tilde{\xi}}\right]\right. \\
& \left.+\frac{1}{\tilde{u}^{2}}\left[\frac{\partial}{\partial \tilde{\xi}}\left(2 \tilde{p}_{e}+2 \tilde{p}_{e} \tilde{u}^{2}-\tilde{\rho}_{e} \tilde{u}-\tilde{\rho}_{e} \tilde{u}^{3}-3 \tilde{p}_{e} \tilde{u}\right)-\tilde{\rho}_{e} \frac{\partial \tilde{\psi}}{\partial \tilde{\xi}}\right]\right\} \\
& +\frac{\partial \tilde{\rho}_{i}}{\frac{m}{m_{e}}} \frac{\partial \tilde{\psi}}{\partial \tilde{\xi}}-\tilde{\rho}_{e} \frac{\partial \tilde{\psi}}{\partial \tilde{\xi}}-\frac{\partial \tilde{\psi}}{\partial \tilde{\xi}} \frac{1}{\tilde{u}^{2}}\left(\frac{m_{e}}{m_{i}}\right)^{2}\left(-\frac{\partial \tilde{\rho}_{i}}{\partial \tilde{\xi}}+\frac{\partial}{\partial \tilde{\xi}}\left(\tilde{\rho}_{i} \tilde{u}\right)\right) \\
& \left.\frac{\partial \tilde{\rho}_{e}}{\partial \tilde{\xi}}+\frac{\partial}{\partial \tilde{\xi}}\left(\tilde{\rho}_{e} \tilde{u}\right)\right)-2 \frac{\partial}{\partial \tilde{\xi}}\left\{\frac{1}{\tilde{u}} \frac{\partial \tilde{\psi}}{\partial \tilde{\xi}}\left[\left(\frac{m_{e}}{m_{i}}\right)^{2} \tilde{\rho}_{i}-\tilde{\rho}_{e}\right]\right\}=0, \\
&
\end{aligned}
$$




$$
\begin{aligned}
& \frac{\partial}{\partial \tilde{\xi}}\left\{\tilde{\rho}_{i} \tilde{u}^{3}+5 \tilde{p}_{i} \tilde{u}-\tilde{\rho}_{i} \tilde{u}^{2}-3 \tilde{p}_{i}\right\}+\frac{\partial}{\partial \xi}\left\{\frac { 1 } { \tilde { u } ^ { 2 } } \frac { m _ { b } } { m _ { i } } \left[\frac { \partial } { \partial \tilde { \xi } } \left(2 \tilde{\rho}_{i} \tilde{u}^{3}+10 \tilde{p}_{i} \tilde{u}\right.\right.\right. \\
& \left.\left.\left.-\tilde{\rho}_{i} \tilde{u}^{4}-8 \tilde{p}_{i} \tilde{u}^{2}-5 \frac{\tilde{p}_{i}^{2}}{\tilde{\rho}_{i}}-\tilde{\rho}_{i} \tilde{u}^{2}-3 \tilde{p}_{i}\right)+\frac{m_{B}}{m_{i}} \frac{\partial \tilde{\psi}}{\partial \tilde{\xi}}\left(2 \tilde{\rho}_{i} \tilde{u}-3 \tilde{\rho}_{i} \tilde{u}^{2}-5 \tilde{p}_{i}\right)\right]\right\} \\
& +2 \frac{m_{b}}{m_{i}} \tilde{\rho}_{i} \tilde{u} \frac{\partial \tilde{\psi}}{\partial \tilde{\xi}}-2 \frac{\partial \tilde{\psi}}{\partial \xi} \frac{1}{\tilde{u}^{2}}\left(\frac{m_{b}}{m_{i}}\right)^{2}\left[\frac{\partial}{\partial \tilde{\xi}}\left(\tilde{\rho}_{i} \tilde{u}^{2}+\tilde{p}_{i}-\tilde{\rho}_{i} \tilde{u}\right)+\tilde{\rho}_{i} \frac{m_{b}}{m_{i}} \frac{\partial \tilde{\psi}}{\partial \tilde{\xi}}\right] \\
& =-\left(\tilde{p}_{i}-\tilde{p}_{\theta}\right) \tilde{u}^{2}\left(1+\frac{m_{i}}{m_{\theta}}\right) \text {, } \\
& \frac{\partial}{\partial \tilde{\xi}}\left\{\tilde{\rho}_{\theta} \tilde{u}^{3}+5 \tilde{p}_{\theta} \tilde{u}-\tilde{\rho}_{\theta} \tilde{u}^{2}-3 \tilde{p}_{\theta}\right\}+\frac{\partial}{\partial \tilde{\xi}}\left\{\frac { 1 } { \tilde { u } ^ { 2 } } \left[\frac { \partial } { \partial \tilde { \xi } } \left(2 \tilde{\rho}_{\theta} \tilde{u}^{3}+10 \tilde{p}_{\theta} \tilde{u}\right.\right.\right. \\
& \left.\left.\left.-\tilde{\rho}_{\theta} \tilde{u}^{4}-8 \tilde{p}_{\theta} \tilde{u}^{2}-5 \frac{\tilde{p}_{\theta}^{2}}{\tilde{\rho}_{\theta}}-\tilde{\rho}_{\theta} \tilde{u}^{2}-3 \tilde{p}_{\theta}\right)+\frac{\partial \tilde{\psi}}{\partial \tilde{\xi}}\left(3 \tilde{\rho}_{\theta} \tilde{u}^{2}+5 \tilde{p}_{\theta}-2 \tilde{\rho}_{\theta} \tilde{u}\right)\right]\right\} \\
& -2 \tilde{\rho}_{\theta} \tilde{u} \frac{\partial \tilde{\psi}}{\partial \tilde{\xi}}+2 \frac{\partial \tilde{\psi}}{\partial \tilde{\xi}} \frac{1}{\tilde{u}^{2}}\left[\frac{\partial}{\partial \tilde{\xi}}\left(\tilde{\rho}_{\theta} \tilde{u}^{2}+\tilde{p}_{\theta}-\tilde{\rho}_{\theta} \tilde{u}\right)-\tilde{\rho}_{\theta} \frac{\partial \tilde{\psi}}{\partial \tilde{\xi}}\right] \\
& =-\left(\tilde{p}_{\theta}-\tilde{p}_{i}\right)\left(1+\frac{m_{i}}{m_{B}}\right) \tilde{u}^{2} \text {. }
\end{aligned}
$$

Some comments to Equations (4.12, 4.15-4.20):

1) Equations. (4.12, 4.15-4.20) contain 6 dependent variables:

$\tilde{\psi}, \tilde{\rho}_{e}, \tilde{\rho}_{i}, \tilde{u}, \tilde{p}_{e}, \tilde{p}_{i}$. Every equation from the system $(4.12,4.15-4.20)$ is of the second order and needs two conditions. The problem belongs to the class of Cauchy problems.

2) In comparison with the Schrödinger theory connected with behavior of the wave function, no special conditions are applied for dependent variables including the domain of the solution existing. This domain is defined automatically in the process of the numerical solution of the concrete variant of calculations.

3) From the introduced scales

$$
u_{0}, \quad x_{0}=\frac{\hbar}{m_{e}} \frac{1}{u_{0}}, \quad \psi_{0}=\frac{m_{e}}{e} u_{0}^{2}, \quad \rho_{0}=\frac{m_{e}^{4}}{4 \pi \hbar^{2} e^{2}} u_{0}^{4}, \quad p_{0}=\rho_{0} u_{0}^{2}=\frac{m_{e}^{4}}{4 \pi \hbar^{2} e^{2}} u_{0}^{6}
$$

only one parameter is independent - the phase velocity $u_{0}$ of the combined quantum object. The value $\hbar / m_{e}=v_{q u}$ can be titled as quantum kinematic viscosity $v_{q u}=1.158 \mathrm{~cm}^{2} / \mathrm{s}$. From this point of view the obtained solutions which will be discussed below have the universal character defined only by Cauchy conditions.

\section{Maple Program Realizing the Solution of the System of Equations (4.15)-(4.20)}

The system of generalized quantum hydrodynamic Equations (4.12), (4.15)-(4.20) have the great possibilities of mathematical modeling as result of changing of twelve Cauchy conditions describing the character features of initial perturbations which lead to the soliton formation.

On this step of investigation we intend to demonstrate the influence of differ- 
ence conditions on the soliton formation. The following figures reflect some results of calculations realized according to the system of Equations (4.12), (4.15)-(4.20) with the help of Maple. The following notations on figures are used: r-density $\tilde{\rho}_{i}, \mathrm{~s}$-density $\tilde{\rho}_{e}, \mathrm{u}$-velocity $\tilde{u}, \mathrm{p}$-pressure $\tilde{p}_{i}, \mathrm{q}$-pressure $\tilde{p}_{e}$ and $\mathrm{v}$-self consistent potential $\tilde{\psi}$. Explanations placed under all following figures, Maple program contains Maple's notations, for example the expression $D(u)(0)=0$ means in usual notations $\frac{\partial \tilde{u}}{\partial \tilde{\xi}}(0)=0$, independent variable $t$ responds to $\tilde{\xi}$.

There is the problem of principle significance-is it possible after a perturbation (defined by Cauchy conditions) to obtain the quantum object of the soliton's kind as result of the self-organization of ionized matter? In the case of the positive answer, what is the origin of existence of this stable object?

Appendixes 1 and 2 contain the corresponding Maple programs. The programs are ready for using.

\section{Super-Conductivity as Movement of Solitons without Destruction}

Let us demonstrate some examples of application of non-local GHE with the help of the Maple program in the Item 5. Significant remarks:

1) We investigate the wave movement of two component ionized matter in the form of moving solitons. It is known that the Schrödinger-Madelung quantum mechanics leads to the soliton destruction.

Extremely important that the really working super-conductivity theory should lead to the soliton conservation in the frame of Cauchy problem, but not as a boundary problem. It means that we should observe the self-organization of matter.

2) The program contains the following Cauchy dimensionless parameters:

$\tilde{\psi}(0)$ - self-consistent potential and $\frac{\partial \tilde{\psi}}{\partial \tilde{\xi}}(0), \tilde{u}(0)$-velocity and $\frac{\partial \tilde{u}}{\partial \tilde{\xi}}(0)$, $\tilde{\rho}_{e}(0)$ density of negative particles and $\frac{\partial \tilde{\rho}_{e}}{\partial \tilde{\xi}}(0), \tilde{\rho}_{i}(0)$ density of positive particles and $\frac{\partial \tilde{\rho}_{i}}{\partial \tilde{\xi}}(0), \quad \tilde{p}_{e}(0)$ pressure of negative particles and $\frac{\partial \tilde{p}_{e}}{\partial \tilde{\xi}}(0)$, $\tilde{p}_{i}(0)$ pressure of positive particles and $\frac{\partial \tilde{p}_{i}}{\partial \tilde{\xi}}(0)$, and the mass ratio of hard $(H)$ and light $(L)$ particles. The program (Appendix 1) is ready for calculations.

3) Let us demonstrate some calculations changing the pressure parameters by ten orders of magnitude. We introduce the dimensionless soliton energetic temperature $\tilde{T}_{e n, s o l}$ using the definition

$$
\tilde{T}_{e n, s o l}=\frac{\tilde{p}_{i}}{\tilde{R}_{i}}
$$

where 


$$
\tilde{R}_{i}=\int_{(\tilde{\xi})} \tilde{\rho}_{i} \mathrm{~d} \tilde{\xi}
$$

Analogically we have

$$
\tilde{S}_{e}=\int_{(\tilde{\xi})} \tilde{\rho}_{e} \mathrm{~d} \tilde{\xi} .
$$

Figures 1-6 reflect the calculations for the Cauchy conditions

$\mathrm{v}(0)=1, \mathrm{r}(0)=1, \mathrm{~s}(0)=1 / 1838, \mathrm{u}(0)=1, \mathrm{p}(0)=1000$,

$\mathrm{q}(0)=950, \mathrm{D}(\mathrm{v})(0)=0, \mathrm{D}(\mathrm{r})(0)=0, \mathrm{D}(\mathrm{s})(0)=0, \mathrm{D}(\mathrm{u})(0)=0$,

$\mathrm{D}(\mathrm{p})(0)=0, \mathrm{D}(\mathrm{q})(0)=0, \mathrm{R}(0)=0, \mathrm{~S}(0)=0$.

Figures 7-12 reflect the calculations for the Cauchy conditions

$\mathrm{v}(0)=1, \mathrm{r}(0)=1, \mathrm{~s}(0)=1 / 1838, \mathrm{u}(0)=1, \mathrm{p}(0)=0.000001$,

$\mathrm{q}(0)=.00000095, \mathrm{D}(\mathrm{v})(0)=0, \mathrm{D}(\mathrm{r})(0)=0, \mathrm{D}(\mathrm{s})(0)=0, \mathrm{D}(\mathrm{u})(0)=0$,

$\mathrm{D}(\mathrm{p})(0)=0, \mathrm{D}(\mathrm{q})(0)=0, \mathrm{R}(0)=0, \mathrm{~S}(0)=0$.

Table 1 contains the Cauchy conditions for pressure, the boundaries $\tilde{\xi}$ (designated as $\lim 1$ and $\lim 2$ ) of the soliton, dimensionless energetic temperature for positive particles and dimensionless concentration of the captured electrons.

From our results obtained in the frame of nonlocal physics follow:

1) Nonlocal quantum hydrodynamics leads to the following physical picture of super-conductivity. So called "quasi-particles" are in reality the solitons which cannot be discovered in Schrödinger-Madelung theory in principal.

$\mathrm{L}=1, \mathrm{H}=1838, \quad \mathrm{R}$-solid line, $\mathrm{S}$-dotted line, $\lim 2=424.77563$

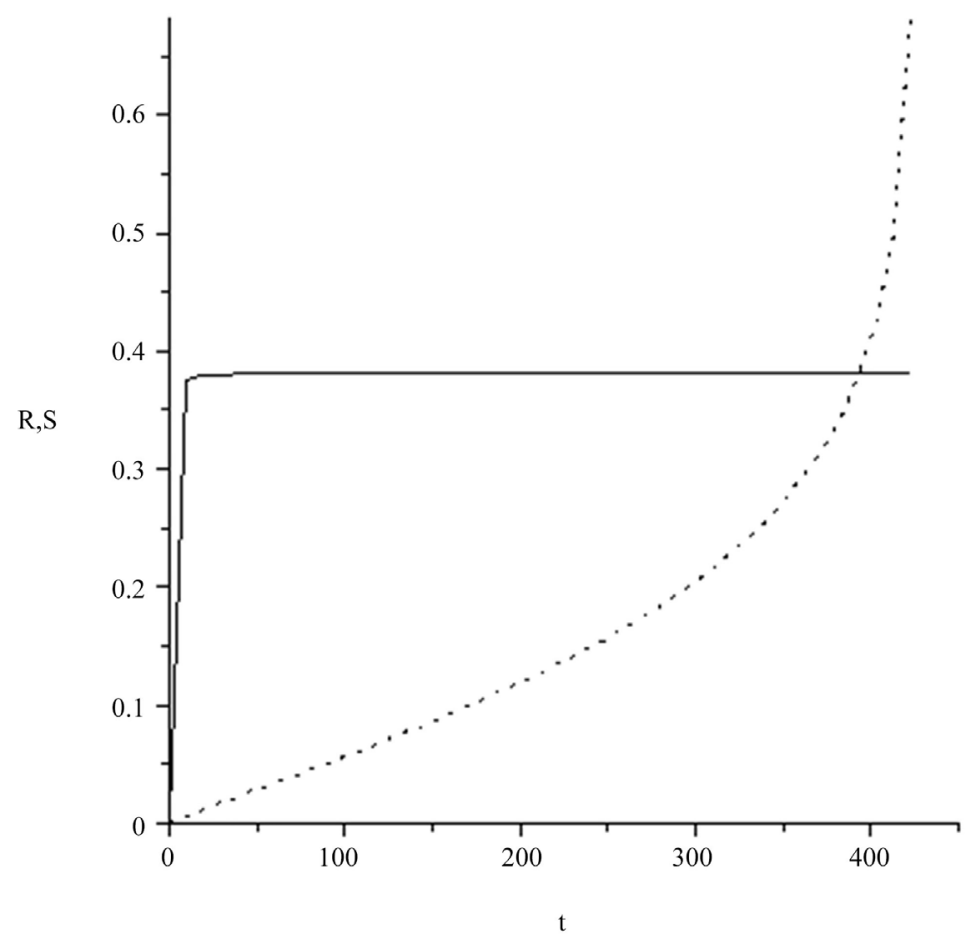

Figure 1. Dimensionless energetic temperature $\mathrm{R}$ for positive particles and dimensionless concentration $\mathrm{S}$ of the captured electrons. 
$\mathrm{L}=1 . \mathrm{H}=1838, \mathrm{p}$-solid line, $\mathrm{q}$-dashed line, $\lim 2=424.77563$

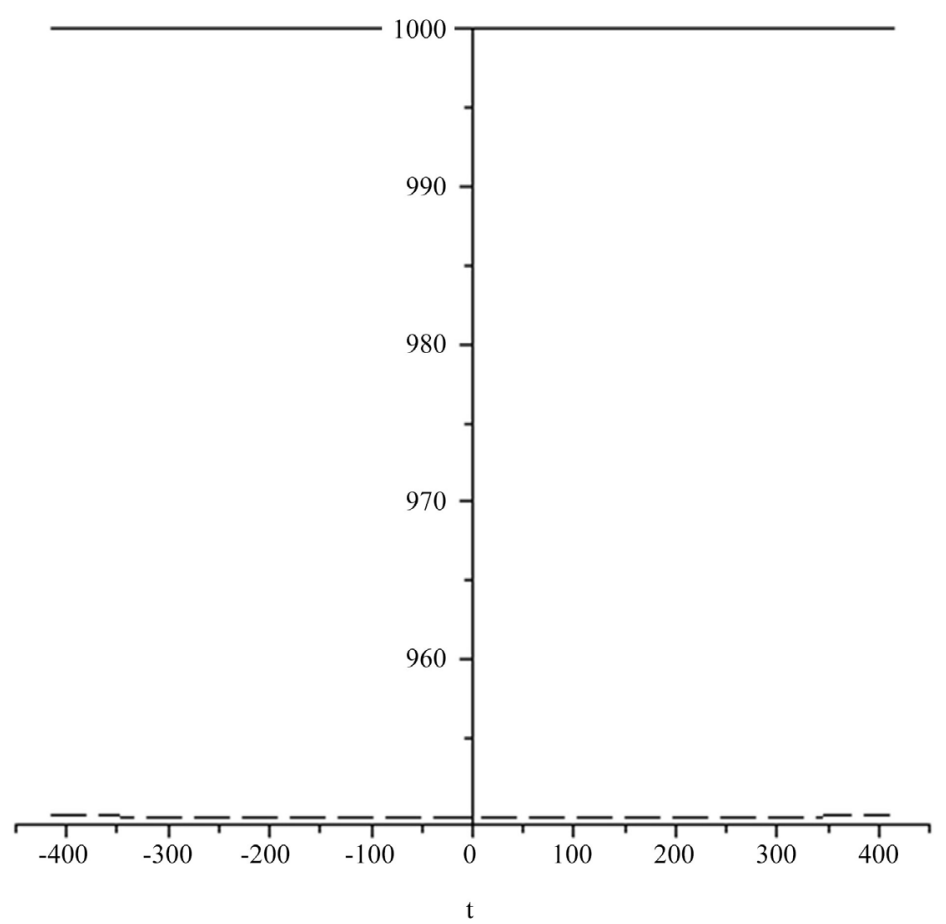

Figure 2. $\mathrm{q}-\tilde{p}_{e}(\tilde{\xi})$ pressure of negative particles, $\mathrm{p}-\tilde{p}_{i}(\tilde{\xi})$ pressure of positive particles.

$\mathrm{L}=1, \mathrm{H}=1838, \quad \mathrm{u}$-solid line, $\mathrm{r}$-dashed line, s-dashdot line, $\lim 2=424.77563$

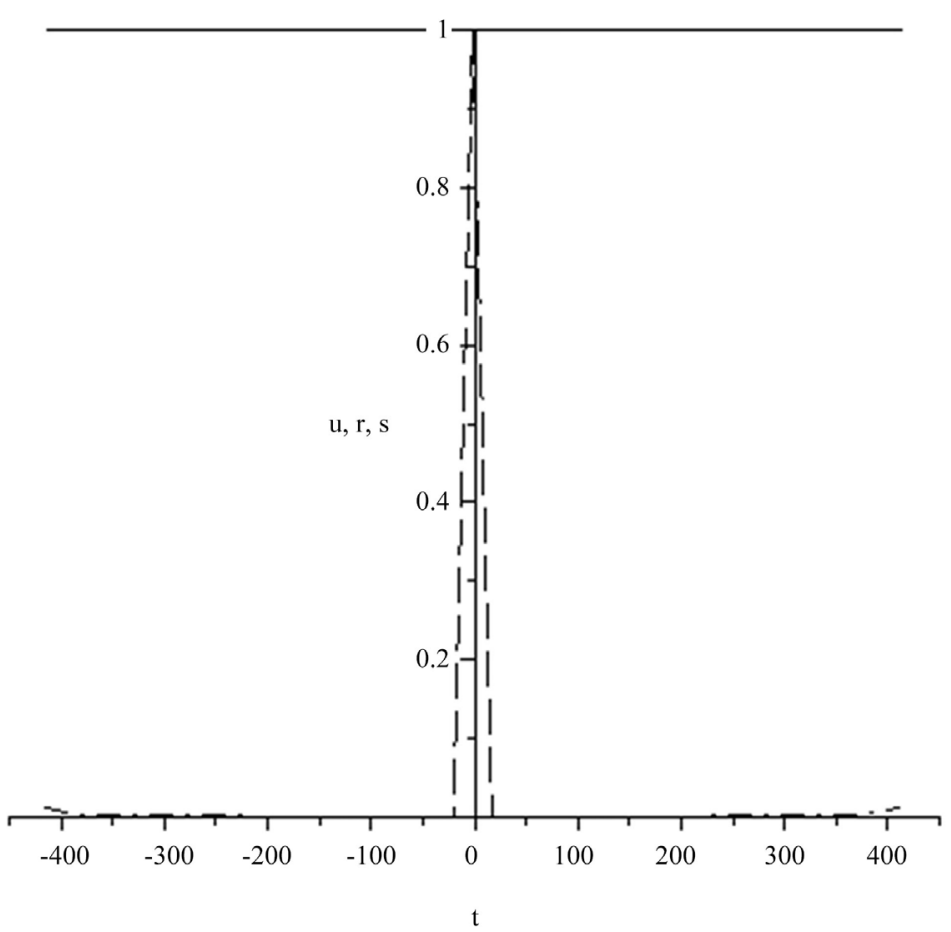

Figure 3. u-velocity $\tilde{u}(\tilde{\xi}), \mathrm{r}$-density $\tilde{\rho}_{i}(\tilde{\xi})$. 
$\mathrm{L}=1, \mathrm{H}=1838, \mathrm{u}-$ solid line, $\lim 1=-424.7756, \lim 2=424.7756$

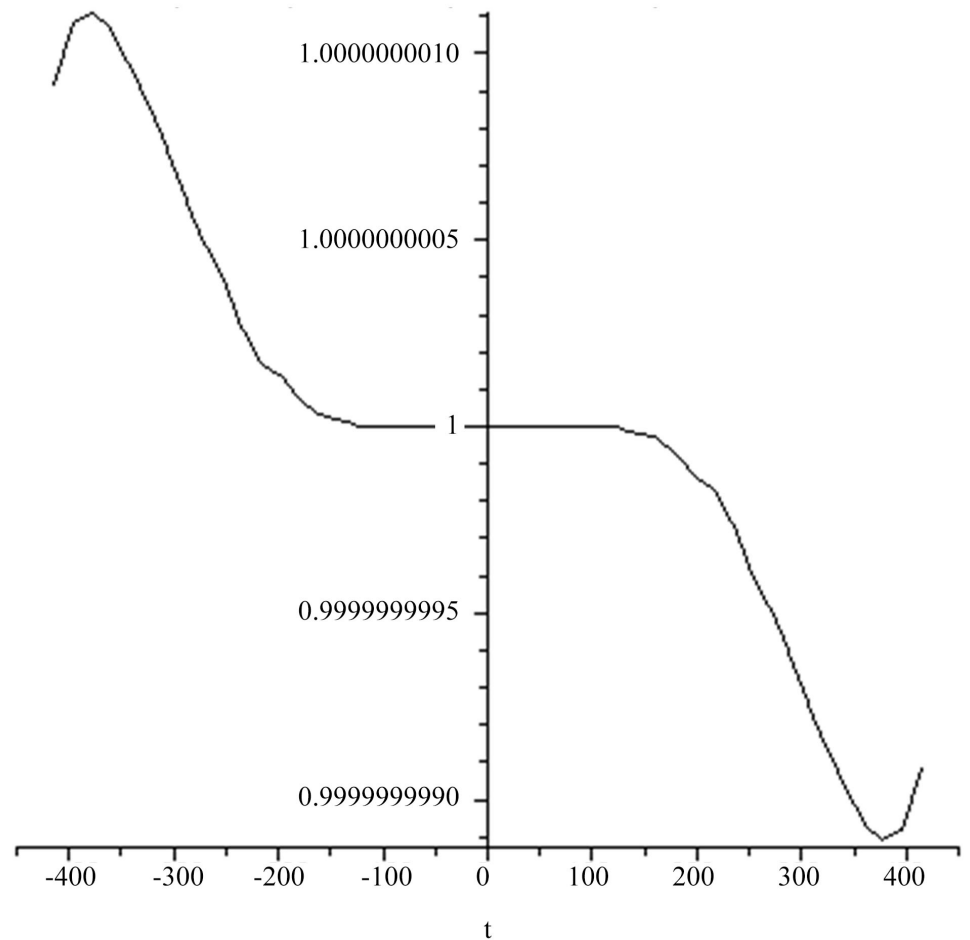

Figure 4. Velocity $\mathrm{u}-\tilde{u}(\xi)$.

$\mathrm{L}=1, \mathrm{H}=1838$, s-solid line, $\lim 2=424.77563$

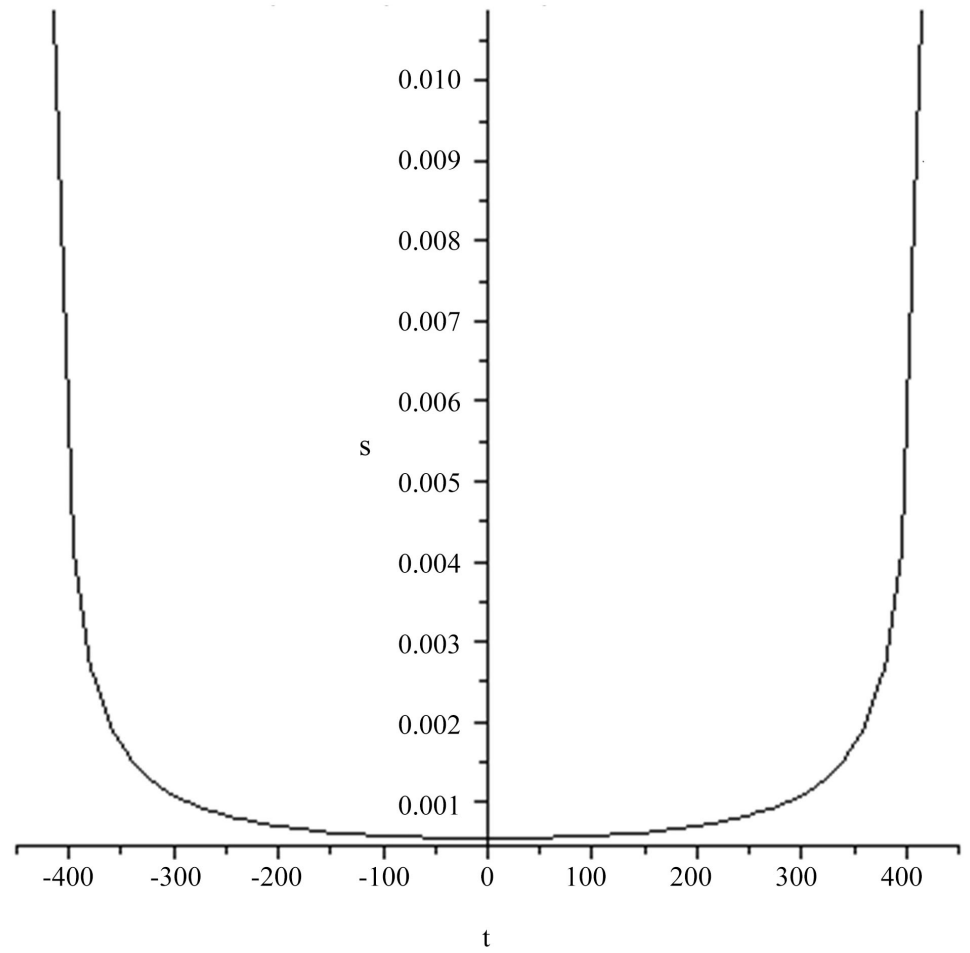

Figure 5. s-density $\tilde{\rho}_{e}(\tilde{\xi})$. 
$\mathrm{L}=1, \mathrm{H}=1838, \mathrm{v}$-solid line, $\lim 2=424.77563$

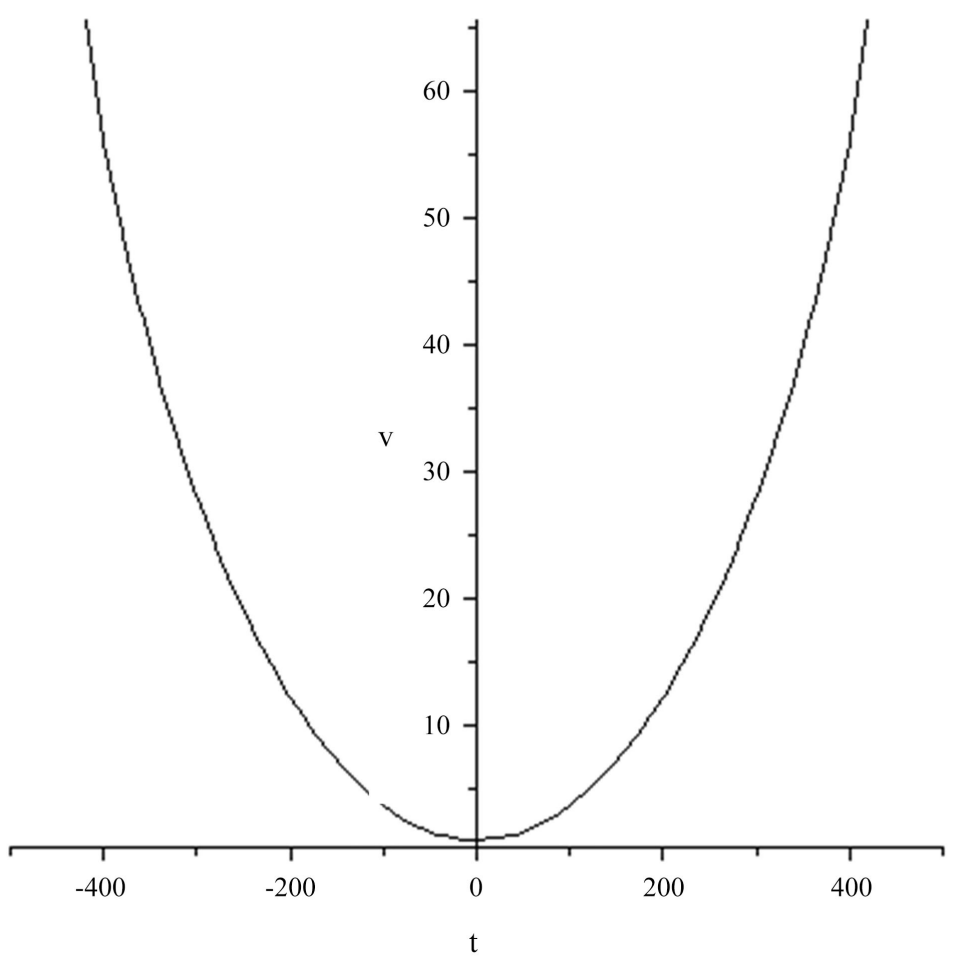

Figure 6. Self-consistent potential $\mathrm{v}-\tilde{\psi}(\tilde{\xi})$.

$\mathrm{L}=1, \mathrm{H}=1838, \mathrm{R}$-solid line, S-dotted line, $\lim 2=0.013432562$

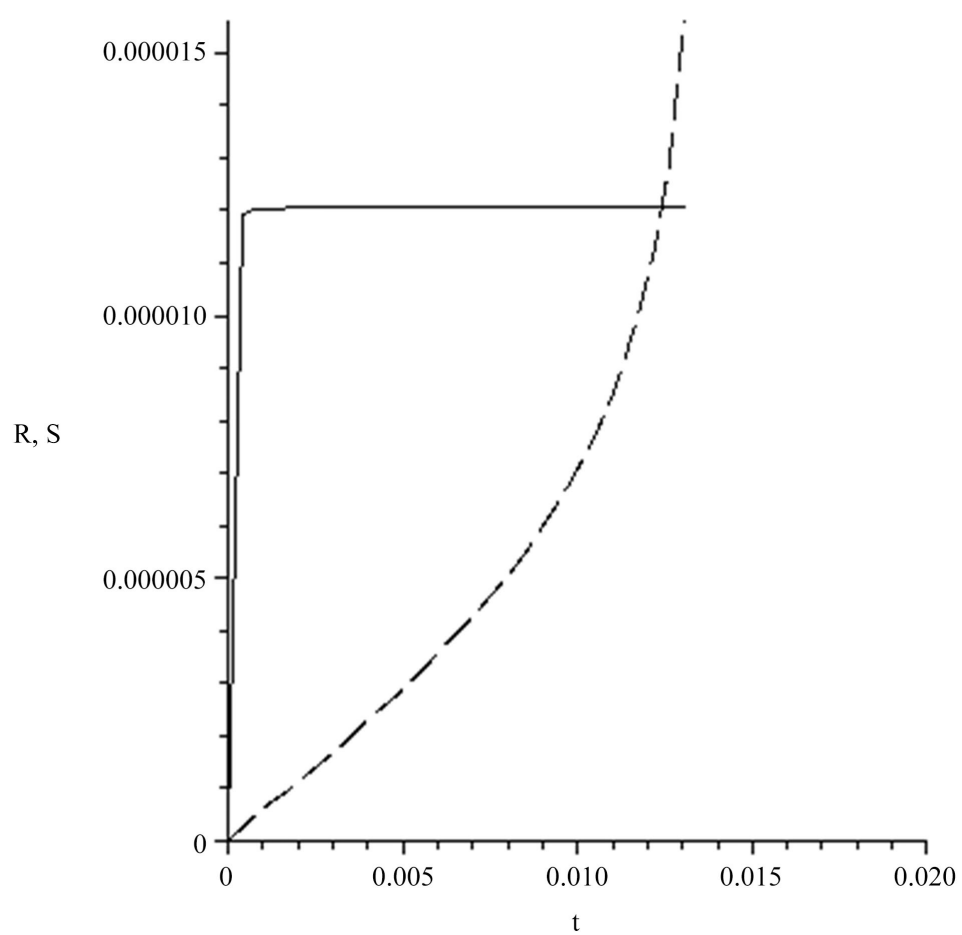

Figure 7. Dimensionless energetic temperature $\mathrm{R}$ for positive particles and dimensionless concentration $\mathrm{S}$ of the captured electrons. 


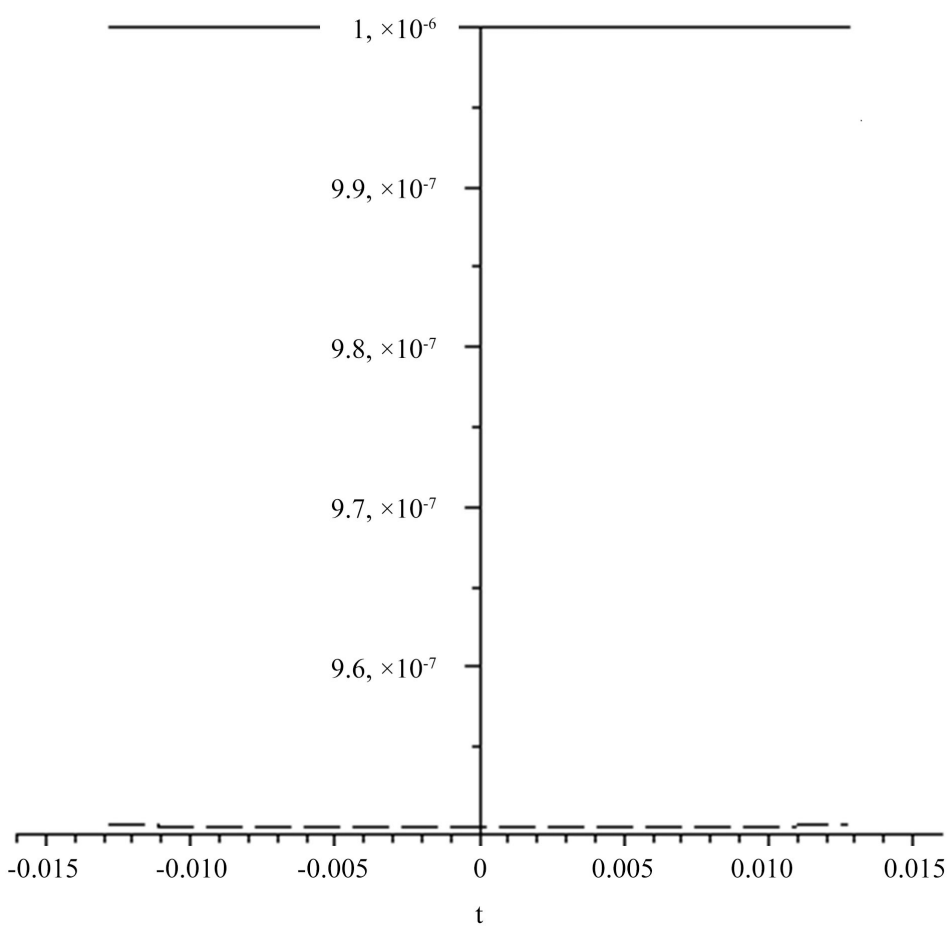

Figure 8. $\mathrm{q}-\tilde{p}_{e}(\tilde{\xi})$ pressure of negative particles, $\mathrm{p}-\tilde{p}_{i}(\tilde{\xi})$ pressure of positive particles.

$\mathrm{L}=1, \mathrm{H}=1838, \mathrm{u}-$ solid line, $\lim 1=-0.01343, \lim 2=0.01343$

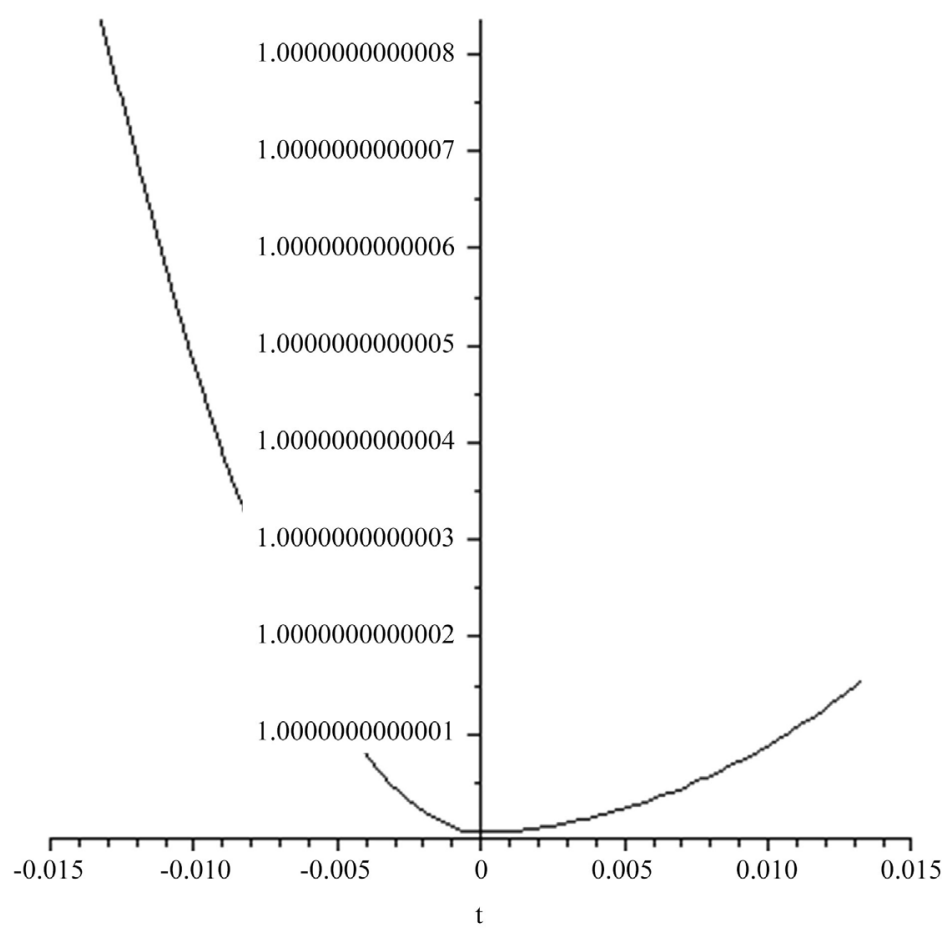

Figure 9. u-velocity $\tilde{u}(\tilde{\xi})$. 
$\mathrm{L}=1, \mathrm{H}=1838, \mathrm{r}$-solid line, $\lim 1=-0.01343, \lim 2=0.01343$

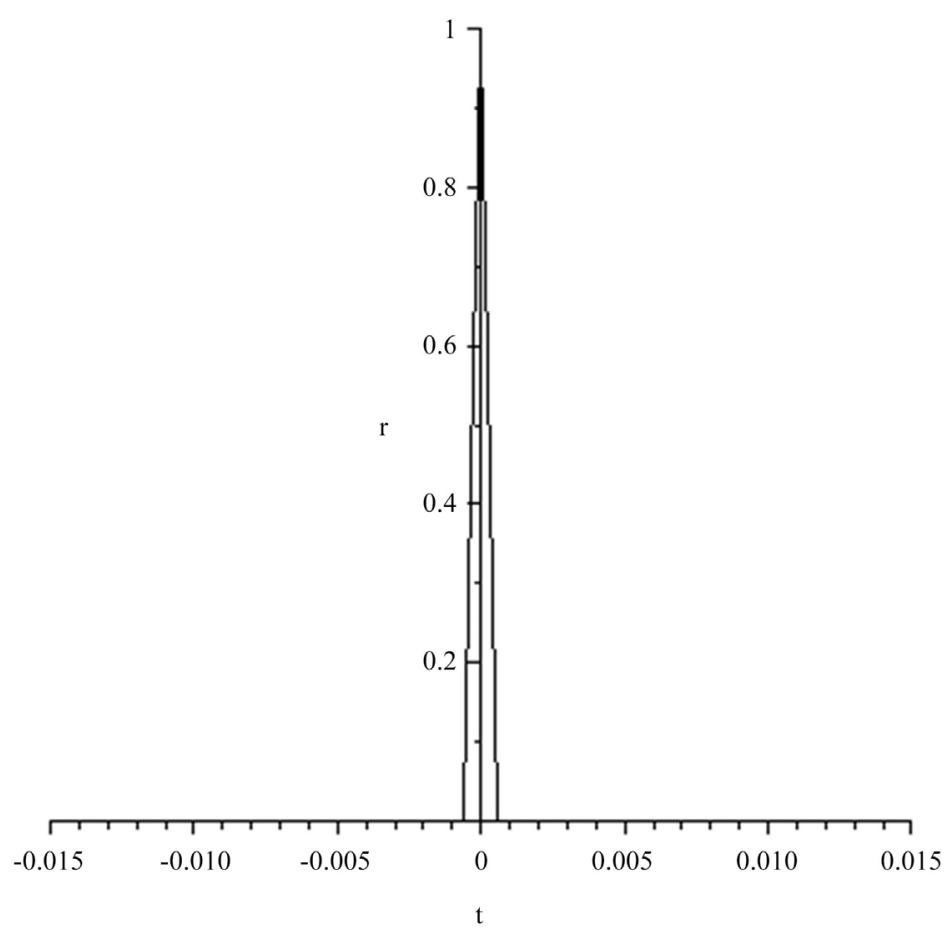

Figure 10. $\mathrm{r}$-density $\tilde{\rho}_{i}(\tilde{\xi})$.

$\mathrm{L}=1, \mathrm{H}=1838$, s-solid line, $\lim 1=-0.01343, \lim 2=0.01343$

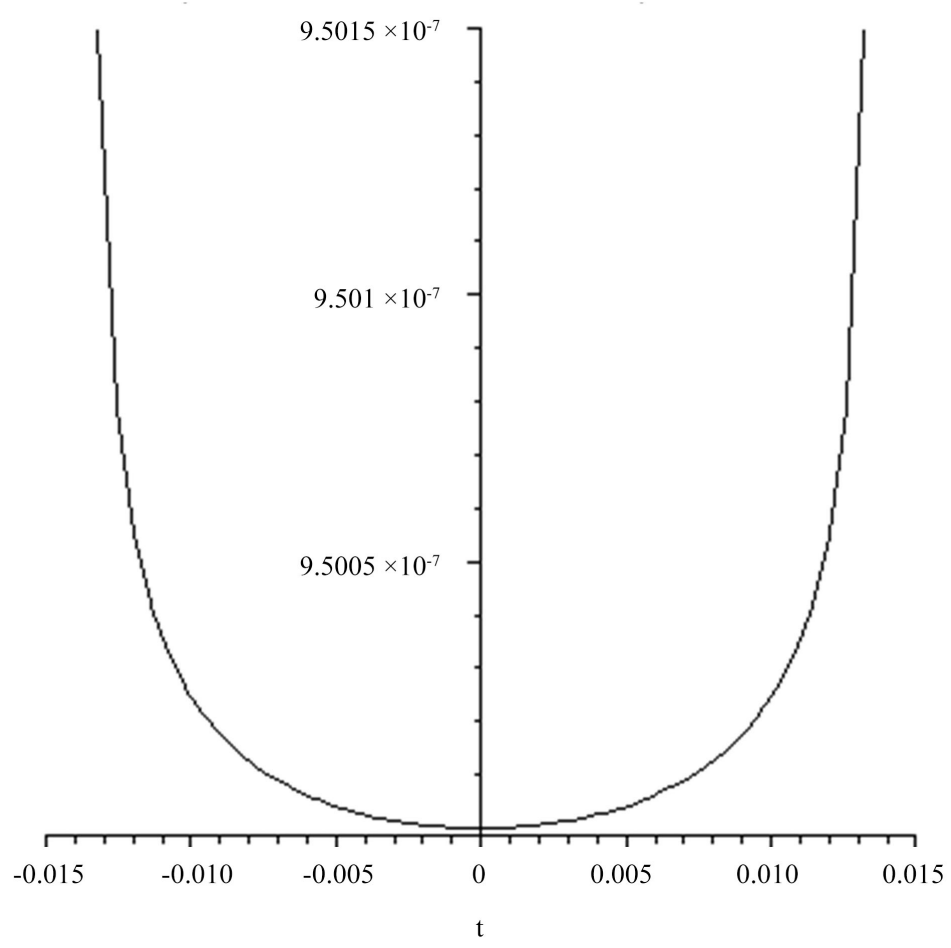

Figure 11. s-density $\tilde{\rho}_{e}(\tilde{\xi})$. 
$\mathrm{L}=1, \mathrm{H}=1838, \mathrm{v}$-solid line, $\lim 1=-0.01343, \lim 2=0.011343$

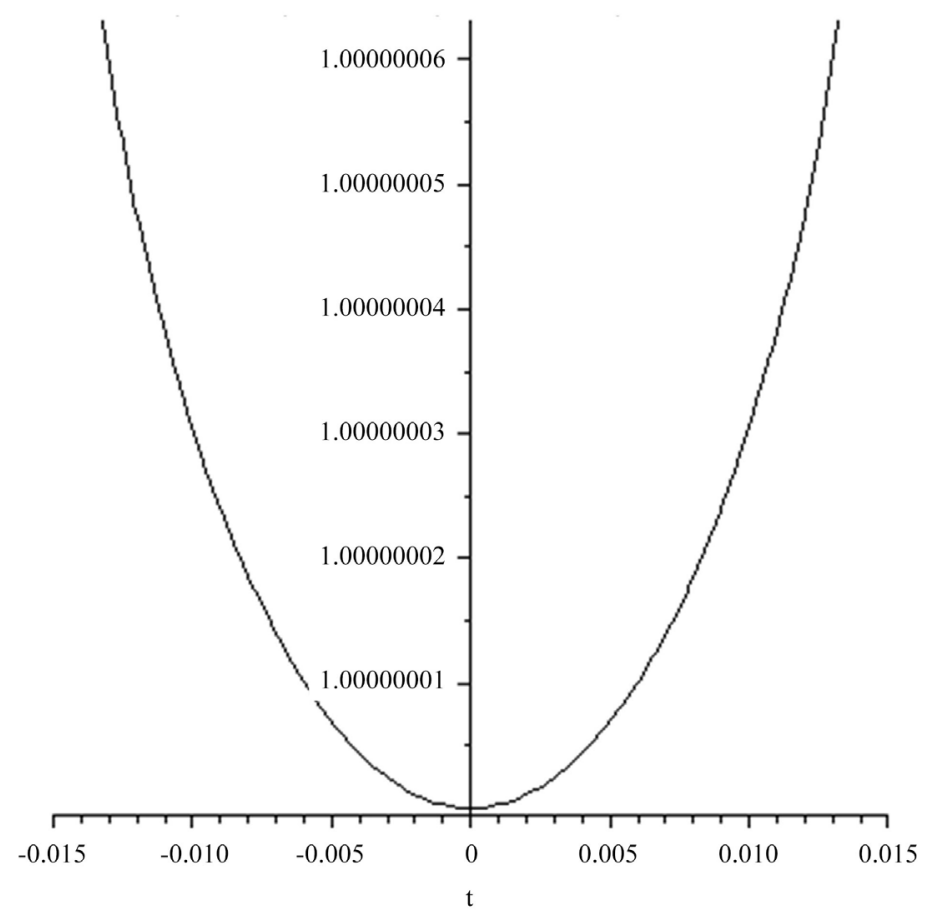

Figure 12. Self-consistent potential v- $\tilde{\psi}(\tilde{\xi})$

Table 1. Cauchy conditions for pressure, the boundaries $\tilde{\xi}$ (designated as $\lim 1$ and $\lim 2$ ) of the soliton, dimensionless energetic temperature for positive particles and dimensionless concentration of the captured electrons.

\begin{tabular}{cccccc}
\hline$\tilde{p}_{i}(0)$ & $\tilde{p}_{e}(0)$ & $-\lim 1$ & $\lim 2$ & $\tilde{T}_{e n, s o l}$ & $\tilde{S}_{e}$ \\
\hline $10^{3}$ & 950 & 424.7759 & 424.7759 & 1309.9075 & 3.8571 \\
$10^{2}$ & 95 & 130.8158 & 130.8158 & 436.4822 & 1.1636 \\
10 & 9.5 & 42.4775 & 42.4775 & 130.9130 & 0.4294 \\
1 & 0.95 & 13.4325 & 13.4325 & 41.3996 & $1.2591 \times 10^{-1}$ \\
0.1 & 0.095 & 4.2477 & 4.2477 & 13.0909 & $4.5475 \times 10^{-2}$ \\
0.01 & 0.0095 & 1.3432 & 1.3432 & 4.1396 & $1.4075 \times 10^{-2}$ \\
$10^{-3}$ & $0.95 \times 10^{-3}$ & 0.4247 & 0.4247 & 1.3090 & $0.4019 \times 10^{-2}$ \\
$10^{-4}$ & $0.95 \times 10^{-4}$ & 0.1343 & 0.1343 & 0.4139 & $1.2488 \times 10^{-3}$ \\
$10^{-5}$ & $0.95 \times 10^{-5}$ & 0.04247 & 0.04247 & 0.1352 & $0.3868 \times 10^{-3}$ \\
$10^{-6}$ & $0.95 \times 10^{-6}$ & 0.01343 & 0.01343 & 0.04139 & $1.0099 \times 10^{-4}$ \\
\hline
\end{tabular}

2) The stability of the quantum object is result of the self organization of the ionized matter. In other words-it is self-consistent influence of electric forces and quantum pressures. Solitons defines the extremely "stiff" structure. Look at Figure 4 and Figure 9-all solitons parts are moving with the same velocity.

3) Stability can be achieved if soliton has negative "shell" and positive "nuclei" and $\tilde{p}_{i}(0)>\tilde{p}_{e}(0)$, or if soliton has positive "shell" and negative "nuclei" and 
$\tilde{p}_{i}(0)<\tilde{p}_{e}(0)$. The moving plane waves remind the structure of "flat atoms" or paired electrons in BCS theory. The previous figures display the typical quantum objects placed in the bounded region of 1D space, all parts of these objects are moving with the same velocity. Namely from calculations follow that in coordinate system moving with the phase velocity, indestructible soliton has the velocity equal to the phase velocity. Moreover the attempt to impose to soliton to move with another group velocity leads to the soliton destruction.

4) Table 1 demonstrates practically the linear dependence between energetic temperature and quantity of captured electrons. All quantum fluctuations in quantum GHE (see Equations 3.1-3.6, 3.7-3.12 and relations 3.15-3.17) are proportional to the Planck constant $\hbar$.

\section{The Charge Carrier's Behavior near the Critical Point}

The step function for profiles in the vicinity of the critical temperature $T_{c}$ can be obtained analytically from the nonlocal GHE equations. Really let us consider the generalized continuum Equations (4.16) and (4.17). Equation

$$
\frac{\partial \tilde{\rho}_{e}}{\partial \tilde{\xi}}-\frac{\partial \tilde{\rho}_{e} \tilde{u}}{\partial \tilde{\xi}}+\frac{\partial}{\partial \tilde{\xi}}\left\{\frac{1}{\tilde{u}^{2}}\left[\frac{\partial}{\partial \tilde{\xi}}\left(\tilde{p}_{e}+\tilde{\rho}_{e}+\tilde{\rho}_{e} \tilde{u}^{2}-2 \tilde{\rho}_{e} \tilde{u}_{e}\right)-\tilde{\rho}_{e} \frac{\partial \tilde{\psi}}{\partial \tilde{\xi}}\right]\right\}=0
$$

can be immediately integrated

$$
\tilde{\rho}_{e}(1-\tilde{u})=-\frac{1}{\tilde{u}^{2}}\left[\frac{\partial}{\partial \tilde{\xi}}\left(\tilde{p}_{e}+\tilde{\rho}_{e}+\tilde{\rho}_{e} \tilde{u}^{2}-2 \tilde{\rho}_{e} \tilde{u}_{e}\right)-\tilde{\rho}_{e} \frac{\partial \tilde{\psi}}{\partial \tilde{\xi}}\right] .
$$

But for all parts of solitons velocity $\tilde{u}=1$. Then

$$
\frac{\partial \tilde{p}_{e}}{\partial \tilde{\xi}}=\tilde{\rho}_{e} \frac{\partial \tilde{\psi}}{\partial \tilde{\xi}} .
$$

In the vicinity of $T_{c}$ we have $\tilde{T}_{e, e n} \sim$ const and Equation (7.3) is written as follows

$$
\tilde{T}_{C, e n, e} \frac{\partial \tilde{\rho}_{e}}{\partial \tilde{\xi}}=\tilde{\rho}_{e} \frac{\partial \tilde{\psi}}{\partial \tilde{\xi}}
$$

or

$$
\tilde{T}_{C, e n, e} \frac{\partial \ln \tilde{\rho}_{e}}{\partial \tilde{\xi}}=\frac{\partial \tilde{\psi}}{\partial \tilde{\xi}} .
$$

After (7.5) integration we have practically step function.

$$
\tilde{\rho}_{e}=\tilde{\rho}_{e 0} \mathrm{e}^{\frac{\tilde{\psi}}{\tilde{T}_{C e, e n}}} .
$$

Analogically for equation

$$
\frac{\partial \tilde{\rho}_{i}}{\partial \tilde{\xi}}-\frac{\partial \tilde{\rho}_{\tilde{i}} \tilde{u}}{\partial \tilde{\xi}}+\frac{m_{e}}{m_{i}} \frac{\partial}{\partial \tilde{\xi}}\left\{\frac{1}{\tilde{u}^{2}}\left[\frac{\partial}{\partial \tilde{\xi}}\left(\tilde{p}_{i}+\tilde{\rho}_{i}+\tilde{\rho}_{i} \tilde{u}^{2}-2 \tilde{\rho}_{i} \tilde{u}_{i}\right)+\frac{m_{e}}{m_{i}} \tilde{\rho}_{i} \frac{\partial \tilde{\psi}}{\partial \tilde{\xi}}\right]\right\}=0
$$

we find

$$
\tilde{\rho}_{i}=\tilde{\rho}_{i 0} \mathrm{e}^{-\frac{m_{e}}{m_{i}} \tilde{\tilde{\psi}}}
$$


Some remarks concerning the scales relations. Let us remind Homes-Zaanen relation [23] [24] [25] [26]

$$
\tau\left(T_{c}\right) \approx \frac{\hbar}{k_{B} T_{c}}
$$

or taking into account that in the soliton's boundaries

$$
\tilde{u}=1
$$

we find (see (3.15))

$$
\tau_{e}=\frac{\hbar}{m_{e} u_{0}^{2}}
$$

where $u_{0}$ is the phase velocity of the soliton's movement. An attempt to impose a velocity different from the phase velocity using Cauchy conditions leads to the destruction of the soliton, and hence to the destruction of superconductivity. Then

$$
m_{e} u_{0}^{2} \sim k_{B} T_{c}
$$

and we reach the scaling law (7.9) in the frame of nonlocal physics

$$
\tau\left(T_{c}\right) \approx \frac{\hbar}{k_{B} T_{c}} .
$$

This time is very short. By analogy with the theory of gravity, it was called the "Planck time" for dissipation. The attempt to tie the superconductivity effects with the cosmological problems seems unacceptable only from the first glance. The origin of this analogy is connected with nonlocal physical kinetics which applicable from the Planck time in the Big Bang theory to the Universe evolution, [7].

It means that we find the phase velocity

$$
u_{0}=\sqrt{\frac{k_{B} T_{c}}{m_{e}}}
$$

or (the value $\hbar / m_{e}=v_{q u}$ can be titled as quantum kinematic viscosity $v_{q u}=1.158 \mathrm{~cm}^{2} / \mathrm{s}$,

Planck constant $\hbar=1.0545726 \times 10^{-34} \mathrm{~J} \cdot \mathrm{s} \quad, \quad$ Boltzmann constant $k_{B}=1.380649 \times 10^{-23} \mathrm{~J} / \mathrm{K}$ )

$u_{0}=\sqrt{v_{q u} \frac{k_{B} T_{c}}{\hbar}}=0.389 \times 10^{6} \sqrt{T_{c}} \frac{\mathrm{cm}}{\mathrm{s}}$, where $T_{c}$ is the temperature measured in Kelvin.

The time scale we used

$$
\tau_{0}=\frac{\hbar}{m_{e} u_{0}^{2}}=\frac{v_{q u}}{u_{0}^{2}}=\frac{\hbar}{k_{B} T_{c}} .
$$

Let us introduce and calculate the corresponding Reynolds number

$$
\operatorname{Re}_{q u}=\frac{u_{0} x_{0}}{v_{q u}}=u_{0} \frac{\hbar}{m_{e} u_{0}} \frac{m_{e}}{\hbar}=1 .
$$


In other words we consider the flow of nonlocal quantum liquid by the condition $\operatorname{Re}_{q u}=1$.

\section{The Generalized Non-Local London's Theory. Longitudinal Electro-Magnetic Waves. The Basic Equations}

We use generalized nonlocal Maxwell equations to prove the existence of longitudinal electromagnetic waves. To this end let's differentiate in time both parts of the Maxwell equation.

$$
\frac{\partial}{\partial t}\left[\frac{\partial}{\partial \mathbf{r}} \times \mathbf{H}\right]=\frac{\partial^{2} \mathbf{D}}{\partial t^{2}}+\frac{\partial}{\partial t} \mathbf{j}^{c}
$$

where $\mathbf{j}^{c}=\mathbf{j}^{a}+\mathbf{j}^{f l}, \quad \mathbf{j}^{f l}$ is fluctuation of the current density. We find

$$
\begin{gathered}
\frac{\partial}{\partial \mathbf{r}} \times \frac{\partial}{\partial t}\left(\mu \mu_{0} \mathbf{H} \frac{1}{\mu \mu_{0}}\right)=\frac{\partial^{2} \mathbf{D}}{\partial t^{2}}+\frac{\partial}{\partial t} \mathbf{j}^{c} \\
\frac{\partial}{\partial \mathbf{r}} \times\left[\frac{1}{\mu \mu_{0}} \frac{\partial}{\partial t}\left[\mu \mu_{0} \mathbf{H}\right]-\mathbf{H} \frac{\partial \ln \mu}{\partial t}\right]=\frac{\partial^{2} \mathbf{D}}{\partial t^{2}}+\frac{\partial}{\partial t} \mathbf{j}^{c} .
\end{gathered}
$$

Obviously the second term in the square bracket can be omitted with the good accuracy, we find

$$
\frac{\partial}{\partial \mathbf{r}} \times\left[\frac{1}{\mu \mu_{0}} \frac{\partial}{\partial t}\left[\mu \mu_{0} \mathbf{H}\right]\right]=\frac{\partial^{2} \mathbf{D}}{\partial t^{2}}+\frac{\partial}{\partial t} \mathbf{j}^{c}
$$

or

$$
\frac{\partial}{\partial \mathbf{r}} \times\left[\frac{1}{\mu \mu_{0}} \frac{\partial}{\partial \mathbf{r}} \times \mathbf{E}\right]=-\frac{\partial^{2} \mathbf{D}}{\partial t^{2}}-\frac{\partial}{\partial t} \mathbf{j}^{c},
$$

It is known that the double vector product is

$$
\mathbf{a} \times \mathbf{b} \times \mathbf{c}=\mathbf{b}(\mathbf{a} \cdot \mathbf{c})-\mathbf{c}(\mathbf{a} \cdot \mathbf{b}) .
$$

Then

$$
\frac{\partial}{\partial \mathbf{r}} \times\left[\frac{1}{\mu \mu_{0}} \frac{\partial}{\partial \mathbf{r}} \times \mathbf{E}\right]=\frac{1}{\mu \mu_{0}} \frac{\partial}{\partial \mathbf{r}}\left[\frac{\partial}{\partial \mathbf{r}} \cdot \mathbf{E}\right]-\frac{1}{\mu \mu_{0}} \Delta \mathbf{E} .
$$

Then the Equation (8.4) is written as follows

$$
\frac{\partial^{2} \mathbf{D}}{\partial t^{2}}+\frac{\partial}{\partial t} \mathbf{j}^{c}=\frac{1}{\mu \mu_{0}} \Delta \mathbf{E}-\frac{1}{\mu \mu_{0}} \frac{\partial}{\partial \mathbf{r}}\left[\frac{\partial}{\partial \mathbf{r}} \cdot \mathbf{E}\right]
$$

or if the electric permeability does not depend on time we have

$$
\varepsilon \varepsilon_{0} \frac{\partial^{2} \mathbf{E}}{\partial t^{2}}+\frac{\partial}{\partial t} \mathbf{j}^{c}=\frac{1}{\mu \mu_{0}}\left\{\Delta \mathbf{E}-\frac{\partial}{\partial \mathbf{r}}\left[\frac{\partial}{\partial \mathbf{r}} \cdot \mathbf{E}\right]\right\}
$$

or

$$
\frac{1}{v_{\phi}^{2}} \frac{\partial^{2} \mathbf{E}}{\partial t^{2}}+\mu \mu_{0} \frac{\partial}{\partial t} \mathbf{j}^{c}=\Delta \mathbf{E}-\frac{\partial}{\partial \mathbf{r}}\left[\frac{\partial}{\partial \mathbf{r}} \cdot \mathbf{E}\right]
$$

where 


$$
\frac{1}{v_{\phi}^{2}}=\varepsilon \varepsilon_{0} \mu \mu_{0}
$$

We write (8.10) in the form

$$
\frac{1}{v_{\phi}^{2}} \frac{\partial^{2} \mathbf{E}}{\partial t^{2}}+\mu \mu_{0} \frac{\partial}{\partial t} \mathbf{j}^{a}+\mu \mu_{0} \frac{\partial}{\partial t} \mathbf{j}^{f l}=\Delta \mathbf{E}-\frac{\partial}{\partial \mathbf{r}}\left[\frac{\partial}{\partial \mathbf{r}} \cdot \mathbf{E}\right]
$$

where (see [4] [8])

$$
\mathbf{j}^{f l}=\tau\left[\frac{\partial}{\partial t}\left(\rho \mathbf{v}_{0}\right)+\frac{\partial}{\partial \mathbf{r}} \cdot\left(\rho \mathbf{v}_{0} \mathbf{v}_{0}\right)+\frac{\partial p}{\partial \mathbf{r}}-\rho \mathbf{g}\right]
$$

or

$$
\mathbf{j}^{f l}=\tau\left[\frac{\partial}{\partial t}\left(n q \mathbf{v}_{0}\right)+\frac{\partial}{\partial \mathbf{r}} \cdot\left(n q \mathbf{v}_{0} \mathbf{v}_{0}\right)+\frac{q}{m} \frac{\partial p}{\partial \mathbf{r}}\left(n k_{B} T\right)-n q \mathbf{g}\right] .
$$

Then the nonlocal Equation (8.12) takes the form

$$
\begin{aligned}
& \frac{1}{v_{\phi}^{2}} \frac{\partial^{2} \mathbf{E}}{\partial t^{2}}+\mu \mu_{0} \frac{\partial}{\partial t}\left\{\tau \frac{\partial}{\partial t} \mathbf{j}^{a}\right\} \\
& =\Delta \mathbf{E}-\frac{\partial}{\partial \mathbf{r}}\left[\frac{\partial}{\partial \mathbf{r}} \cdot \mathbf{E}\right]-\mu \mu_{0} \frac{\partial}{\partial t} \mathbf{j}^{a} \\
& \quad-\mu \mu_{0} \frac{\partial}{\partial t}\left\{\tau\left[\frac{\partial}{\partial \mathbf{r}} \cdot\left(\mathbf{j}^{a} \mathbf{v}_{0}\right)+\frac{q}{m} \frac{\partial}{\partial \mathbf{r}}\left(n k_{B} T\right)-n q \mathbf{g}\right]\right\} .
\end{aligned}
$$

Let be

$$
\mathbf{j}^{a}=\sigma \mathbf{E},
$$

where $\sigma$ is the coefficient of conductivity. In the simplest Drude model

$$
\sigma=\frac{n e^{2}}{2 m^{*}} \tau_{r},
$$

where $n$ is numerical electron density, $m^{*}$ is effective mass, $\tau_{r}$ is the relaxation time, the dimension of the conductivity is $\mathrm{s}^{-1}$. As we see from the definition of $m^{*}$, the introduction of an "effective" mass translates the relaxation time to the level of the fitting parameter. If nonlocal parameter $\tau=$ const and the coefficient of conductivity $\sigma=\sigma(x, y, z)$, we have

$$
\begin{aligned}
& \frac{\partial^{2} \mathbf{E}}{\partial t^{2}}+\mu \mu_{0} v_{\phi}^{2} \tau \frac{\partial^{2} \mathbf{j}^{a}}{\partial t^{2}} \\
= & v_{\phi}^{2}\left\{\Delta \mathbf{E}-\frac{\partial}{\partial \mathbf{r}}\left[\frac{\partial}{\partial \mathbf{r}} \cdot \mathbf{E}\right]\right\}-\mu \mu_{0} v_{\phi}^{2} \frac{\partial}{\partial t} \mathbf{j}^{a} \\
& -\mu \mu_{0} v_{\phi}^{2} \tau \frac{\partial}{\partial t}\left[\frac{\partial}{\partial \mathbf{r}} \cdot\left(\mathbf{j}^{a} \mathbf{v}_{0}\right)\right]-\mu \mu_{0} v_{\phi}^{2} \tau \frac{\partial}{\partial t}\left[\frac{q}{m} \frac{\partial}{\partial \mathbf{r}}\left(n k_{B} T\right)-n q \mathbf{g}\right]
\end{aligned}
$$

or

$$
\begin{aligned}
{\left[1+\frac{\tau \sigma}{\varepsilon_{0} \varepsilon}\right] \frac{\partial^{2} \mathbf{E}}{\partial t^{2}}=} & v_{\varphi}^{2}\left\{\Delta \mathbf{E}-\frac{\partial}{\partial \mathbf{r}}\left[\frac{\partial}{\partial \mathbf{r}} \cdot \mathbf{E}\right]\right\}-\frac{\sigma}{\varepsilon_{0} \varepsilon} \frac{\partial \mathbf{E}}{\partial t}-\frac{\tau}{\varepsilon_{0} \varepsilon} \frac{\partial}{\partial t}\left[\frac{\partial}{\partial \mathbf{r}} \cdot\left(\sigma \mathbf{E v}_{0}\right)\right] \\
& -\frac{\tau}{\varepsilon_{0} \varepsilon} \frac{\partial}{\partial t}\left[\frac{q}{m} \frac{\partial}{\partial \mathbf{r}}\left(n k_{B} T\right)-n q \mathbf{g}\right]
\end{aligned}
$$


Equation (8.19) is the basement relation for following investigation.

\section{The Nonlocal Theory of Longitudinal Electromagnetic Waves. The 1D Non-Stationary Case}

Let us investigate the 1D non-stationary case. For the 1D case ( $x$-direction), we find from (8.19)

$$
\begin{aligned}
{\left[1+\frac{\tau \sigma}{\varepsilon_{0} \varepsilon}\right] \frac{\partial^{2} E_{x}}{\partial t^{2}}=} & -\frac{\sigma}{\varepsilon_{0} \varepsilon} \frac{\partial E_{x}}{\partial t}-\frac{\tau}{\varepsilon_{0} \varepsilon} \frac{\partial}{\partial t}\left[\frac{\partial}{\partial x}\left(\sigma E_{x} v_{0 x}\right)\right] \\
& -\frac{\tau}{\varepsilon_{0} \varepsilon} \frac{\partial}{\partial t}\left[\frac{q}{m} \frac{\partial}{\partial x}\left(n k_{B} T\right)-n q g_{x}\right] .
\end{aligned}
$$

After integration we find

$$
\begin{aligned}
{\left[1+\frac{\tau \sigma}{\varepsilon_{0} \varepsilon}\right] \frac{\partial E_{x}}{\partial t}=} & -\frac{\sigma}{\varepsilon_{0} \varepsilon} E_{x}-\frac{\tau}{\varepsilon_{0} \varepsilon} \frac{\partial}{\partial x}\left(\sigma E_{x} v_{0 x}\right) \\
& -\frac{\tau}{\varepsilon_{0} \varepsilon} q\left[\frac{1}{m} \frac{\partial}{\partial x}\left(n k_{B} T\right)-n g_{x}\right]+f(x),
\end{aligned}
$$

where the Boltzmann constant $k_{B}=1.380649 \times 10^{-23} \mathrm{~J} / \mathrm{K}$.

Let us consider the main features of the solution of Equation (9.2). With this aim we suppose that $v_{0 x}=$ const,$\sigma=$ const and the last term of the equation can be omitted. The penultimate term in Equation (9.2) can be omitted if the pressure gradient can compensate the influence of the gravitation force acting on the volume unit and $f(x)=0$. We have

$$
\left[1+\frac{\tau \sigma}{\varepsilon_{0} \varepsilon}\right] \frac{\partial E_{x}}{\partial t}+\frac{\tau \sigma v_{0 x}}{\varepsilon_{0} \varepsilon} \frac{\partial E_{x}}{\partial x}=-\frac{\sigma}{\varepsilon_{0} \varepsilon} E_{x} .
$$

The solution of this equation is a damping longitudinal E-wave. Really

$$
E_{x}=\exp \left(-\frac{1}{\tau v_{0 x}} x\right) \Phi\left[\left(1+\frac{\tau \sigma}{\varepsilon_{0} \varepsilon}\right) x-\frac{\tau \sigma v_{0 x}}{\varepsilon_{0} \varepsilon} t\right] .
$$

Conclusions:

1) The validity of the solution (9.4) could be verified by the direct substitution of this relation (9.4) into Equation (9.3).

2) If the non-locality parameter $\tau$ is equal to zero, the electric intensity for the longitudinal waves (EMLW) also turns into zero. It means that EMLW cannot exist in the frame of the classic Maxwell electrodynamics.

Equation (9.2) can be written in the form

$$
\left[1+\frac{\tau \sigma}{\varepsilon_{0} \varepsilon}\right] \frac{\partial E_{x}}{\partial t}+\frac{\tau \sigma}{\varepsilon_{0} \varepsilon} v_{0 x} \frac{\partial E_{x}}{\partial x}=-\frac{\sigma}{\varepsilon_{0} \varepsilon} E_{x}-\frac{\tau}{\varepsilon_{0} \varepsilon} \frac{q}{m}\left[\frac{\partial}{\partial x}\left(n k_{B} T\right)-\rho g_{x}\right] .
$$

The last term in square bracket takes into account the influence of the pressure gradient and the gravitation force. If nonlocal parameter $\tau=0$, then

$$
\frac{\partial E_{x}}{\partial t}=-\frac{\sigma}{\varepsilon_{0} \varepsilon} E_{x}
$$

and we have in the local electrodynamics the exponential $E_{x}$ attenuation (if 
$f(x)=0$ in (9.2)) without the wave creation

$$
E_{x}=E_{x, t=0} \exp \left(-\frac{\sigma t}{\varepsilon \varepsilon_{0}}\right) .
$$

The existence of longitudinal electromagnetic waves does not contradict (nonlocal) electrodynamics if longitudinal waves are actually detected and the medium in which they are generated is known. Then in this case the problem of substantiating such waves in electrodynamics is reduced to the search for material equations characterizing the response of the medium to the influence of the field and the joint solution of these material equations and Maxwell's equations, with appropriate boundary conditions.

Let be

$$
\left(1+\frac{\tau \sigma}{\varepsilon_{0} \varepsilon}\right) x-\frac{\tau \sigma v_{0}}{\varepsilon_{0} \varepsilon} t=\text { const }=C .
$$

Then

$$
\left(1+\frac{\tau \sigma}{\varepsilon_{0} \varepsilon}\right) \frac{\mathrm{d} x}{\mathrm{~d} t}=\frac{\tau \sigma v_{0}}{\varepsilon_{0} \varepsilon}
$$

or

$$
v_{E M L W}=\frac{\mathrm{d} x}{\mathrm{~d} t}=\frac{\tau \sigma v_{0}}{\varepsilon_{0} \varepsilon}\left[1+\frac{\tau \sigma}{\varepsilon_{0} \varepsilon}\right]^{-1}=\frac{\tau \sigma v_{0}}{\varepsilon_{0} \varepsilon} \frac{\varepsilon_{0} \varepsilon}{\varepsilon_{0} \varepsilon+\tau \sigma}=\frac{\tau \sigma}{\varepsilon_{0} \varepsilon+\tau \sigma} v_{0}=\frac{1}{1+\frac{\varepsilon_{0} \varepsilon}{\tau \sigma}} v_{0} .
$$

Low permittivity (low epsilon) materials are now attracting wide attention due to potential novel applications in optics and radio communications. Surface plasmon polaritons (SPPs) are electromagnetic waves that travel along a metal-dielectric or metal-air interface, practically in the infrared or visible-frequency. The term "surface plasmon polariton" explains that the wave involves both charge motion in the metal ("surface plasmon") and electromagnetic waves in the air or dielectric ("polariton"). Application of SPPs enables subwavelength optics in microscopy and lithography beyond the diffraction limit. It also enables the first steady-state micro-mechanical measurement of a fundamental property of light itself: the momentum of a photon in a dielectric medium.

Longitudinal electromagnetic waves play important role in plasma, in surface plasmon polaritons in anisotropic materials, in space-charge waves in semiconductor materials. Then for so called that in Epsilon Near Zero (ENZ) materials the velocity $v_{E M L W} \cong v_{0}$ (the usual light velocity). For the following details of the wave process one needs the explicit form of the $\Phi(x, t)$ function and boundary and initial conditions. As we see from (9.10)

$$
v_{E M L W} \rightarrow v_{0} \text { if } \varepsilon \rightarrow 0 .
$$

Obviously to overcome this velocity limit is possible only in the systems with negative conductivity where formally

$$
v_{E M L W} \rightarrow \infty \text { if } \sigma \rightarrow-\frac{\varepsilon_{0} \varepsilon}{\tau} .
$$


The possibility of the appearance of negative conductivity in a non-equilibrium electron system, i.e., a situation in which the current flows opposite to the electric field, was apparently indicated for the first time by Krömer in the late 1950s [27]. The mechanism of absolute negative conductivity (ANC) in a two-dimensional electron system placed into magnetic and ac electric fields, which is associated with two-dimensional electron scattering by impurities, accompanied by ac field photon absorption, was proposed in [28] [29]. The state with negative conductivity is unstable, the system decays into domains, and the measured macroscopic resistance becomes zero. The existence of this effect was experimentally confirmed in 2002 [30].

\section{Non-Local Magnetic Field Evolution in Plasma}

We consider the 3D non-stationary magnetic field evolution in plasma. With this aim let us transform Equation (8.19) written as follows

$$
\begin{aligned}
{\left[1+\frac{\tau \sigma}{\varepsilon_{0} \varepsilon}\right] \frac{\partial^{2} \mathbf{E}}{\partial t^{2}}=} & v_{\phi}^{2}\left\{\Delta \mathbf{E}-\frac{\partial}{\partial \mathbf{r}}\left[\frac{\partial}{\partial \mathbf{r}} \cdot \mathbf{E}\right]\right\}-\frac{\sigma}{\varepsilon_{0} \varepsilon} \frac{\partial \mathbf{E}}{\partial t}-\frac{\tau}{\varepsilon_{0} \varepsilon} \frac{\partial}{\partial t}\left[\frac{\partial}{\partial \mathbf{r}} \cdot\left(\sigma \mathbf{E v}_{0}\right)\right] \\
& -\frac{\tau}{\varepsilon_{0} \varepsilon} \frac{\partial}{\partial t}\left[\frac{q}{m} \frac{\partial}{\partial \mathbf{r}}\left(n k_{B} T\right)-n q \mathbf{g}\right]
\end{aligned}
$$

Let us apply the vector product operator to the left and right sides of (10.1)

$$
\begin{aligned}
{\left[1+\frac{\tau \sigma}{\varepsilon_{0} \varepsilon}\right] \frac{\partial^{2}}{\partial t^{2}} \frac{\partial}{\partial \mathbf{r}} \times \mathbf{E}=} & v_{\phi}^{2} \frac{\partial}{\partial \mathbf{r}} \times\left\{\Delta \mathbf{E}-\frac{\partial}{\partial \mathbf{r}}\left[\frac{\partial}{\partial \mathbf{r}} \cdot \mathbf{E}\right]\right\}-\frac{\sigma}{\varepsilon_{0} \varepsilon} \frac{\partial}{\partial t} \frac{\partial}{\partial \mathbf{r}} \times \mathbf{E} \\
& -\frac{\tau}{\varepsilon_{0} \varepsilon} \frac{\partial}{\partial t} \frac{\partial}{\partial \mathbf{r}} \times\left[\frac{\partial}{\partial \mathbf{r}} \cdot\left(\sigma \mathbf{E v}_{0}\right)\right]+\frac{\tau}{\varepsilon_{0} \varepsilon} \frac{\partial}{\partial t} \frac{\partial}{\partial \mathbf{r}} \times[n q \mathbf{g}] .
\end{aligned}
$$

Let us remind that in the coordinate notations we have

$$
\left[\frac{\partial}{\partial \mathbf{r}} \cdot\left(\sigma \mathbf{E} \mathbf{v}_{0}\right)\right]=\frac{\partial}{\partial x}\left(\sigma E_{x} \mathbf{v}_{0}\right)+\frac{\partial}{\partial y}\left(\sigma E_{y} \mathbf{v}_{0}\right)+\frac{\partial}{\partial z}\left(\sigma E_{z} \mathbf{v}_{0}\right),
$$

We use $e$ as absolute charge of electron, $\mathbf{v}_{0}$ as a mean velocity of the electron motion and the operator relation

$$
\frac{\partial}{\partial \mathbf{r}} \times\left[\frac{\partial p}{\partial \mathbf{r}}\right] \equiv 0
$$

The corresponding current density is

$$
\mathbf{j}_{0}=-n_{e} e \mathbf{v}_{0}
$$

We introduce also the charge number density

$$
\rho_{e}=e n_{e}
$$

and the character relaxation time

$$
\tau_{r}=\frac{\varepsilon_{0} \varepsilon}{\sigma} .
$$

We transform (10.2):

1) Keeping only the terms proportional to the first power of the non-local parameter $\tau$, 
2) Using the relations (for simplicity) $\varepsilon=$ const, $\sigma=$ const ; the following transformation should be taken into account

$$
\begin{aligned}
\frac{\tau}{\varepsilon_{0} \varepsilon} \frac{\partial}{\partial \mathbf{r}} \times\left[\frac{\partial}{\partial \mathbf{r}} \cdot\left(\sigma \mathbf{E v}_{0}\right)\right] & =\frac{\tau \sigma}{\varepsilon_{0} \varepsilon} \frac{\partial}{\partial \mathbf{r}} \times\left[\frac{\partial}{\partial x}\left(E_{x} \mathbf{v}_{0}\right)+\frac{\partial}{\partial y}\left(E_{y} \mathbf{v}_{0}\right)+\frac{\partial}{\partial z}\left(E_{z} \mathbf{v}_{0}\right)\right] \\
& =\frac{\tau \sigma}{\varepsilon_{0} \varepsilon} \frac{\partial}{\partial \mathbf{r}} \times\left[\mathbf{v}_{0} \frac{\partial}{\partial \mathbf{r}} \cdot \mathbf{E}\right]+\frac{\tau \sigma}{\varepsilon_{0} \varepsilon} \frac{\partial}{\partial \mathbf{r}} \times\left(\mathbf{E} \cdot \frac{\partial}{\partial \mathbf{r}}\right) \mathbf{v}_{0} \\
& =\frac{\tau \sigma}{\varepsilon_{0} \varepsilon} \frac{1}{\varepsilon_{0} \varepsilon} \frac{\partial}{\partial \mathbf{r}} \times\left[\mathbf{v}_{0} \frac{\partial}{\partial \mathbf{r}} \cdot \mathbf{D}\right]+\frac{\tau \sigma}{\varepsilon_{0} \varepsilon} \frac{\partial}{\partial \mathbf{r}} \times\left(\mathbf{E} \cdot \frac{\partial}{\partial \mathbf{r}}\right) \mathbf{v}_{0} \\
& =-\frac{\tau \sigma}{\varepsilon_{0} \varepsilon} \frac{\partial}{\partial \mathbf{r}} \times \mathbf{j}_{0 e}+\frac{\tau \sigma}{\varepsilon_{0} \varepsilon} \frac{\partial}{\partial \mathbf{r}} \times\left(\mathbf{E} \cdot \frac{\partial}{\partial \mathbf{r}}\right) \mathbf{v}_{0 e} .
\end{aligned}
$$

The last line in (10.8) corresponds the physical system in which the charge current is realized by electrons. Extremely important that the last term in (10.8) contains the hydrodynamic velocity $\mathbf{v}_{0 e}$, which can be defined only as a result of the whole hydrodynamic problem.

Then if we want to separate the electro-dynamic and hydro-dynamic problems we should neglect the changes in the space of the hydrodynamic velocity fluctuations. Therefore we use the relation

$$
\frac{\tau}{\varepsilon_{0} \varepsilon} \frac{\partial}{\partial \mathbf{r}} \times\left[\frac{\partial}{\partial \mathbf{r}} \cdot\left(\sigma \mathbf{E v}_{0}\right)\right]=-\frac{\tau \sigma}{\varepsilon_{0} \varepsilon} \frac{\partial}{\partial \mathbf{r}} \times \mathbf{j}_{0 e} .
$$

Using also the Maxwell relation

$$
\frac{\partial}{\partial \mathbf{r}} \times \mathbf{E}=-\frac{\partial \mathbf{B}}{\partial t}
$$

we write (10.2) as follows (omitting the gravitation influence)

$$
-\left[1+\frac{\tau}{\tau_{r}}\right] \frac{\partial^{2}}{\partial t^{2}} \frac{\partial \mathbf{B}}{\partial t}=v_{\phi}^{2} \frac{\partial}{\partial \mathbf{r}} \times\left\{\Delta \mathbf{E}-\frac{\partial}{\partial \mathbf{r}}\left[\frac{\partial}{\partial \mathbf{r}} \cdot \mathbf{E}\right]\right\}+\frac{1}{\tau_{r}} \frac{\partial}{\partial t} \frac{\partial \mathbf{B}}{\partial t}+\frac{\tau}{\tau_{r}} \frac{1}{\varepsilon \varepsilon_{0}} \frac{\partial}{\partial t} \frac{\partial}{\partial \mathbf{r}} \times \mathbf{j}_{0} .
$$

Keeping as before only the terms proportional to the first power of the non-local parameter $\tau$, we can use the Maxwell relation

$$
\mathbf{j}_{0}+\frac{\partial \mathbf{D}}{\partial t}=\frac{\partial}{\partial \mathbf{r}} \times \mathbf{H}
$$

Then

$$
\begin{aligned}
-\left[1+\frac{\tau \sigma}{\varepsilon_{0} \varepsilon}\right] \frac{\partial^{2}}{\partial t^{2}} \frac{\partial \mathbf{B}}{\partial t}= & v_{\phi}^{2} \frac{\partial}{\partial \mathbf{r}} \times\left\{\Delta \mathbf{E}-\frac{\partial}{\partial \mathbf{r}}\left[\frac{\partial}{\partial \mathbf{r}} \cdot \mathbf{E}\right]\right\}+\frac{\sigma}{\varepsilon_{0} \varepsilon} \frac{\partial}{\partial t} \frac{\partial \mathbf{B}}{\partial t} \\
& +\frac{\tau}{\tau_{r}} \frac{1}{\varepsilon \varepsilon_{0}} \frac{\partial}{\partial t} \frac{\partial}{\partial \mathbf{r}} \times\left\{\frac{\partial}{\partial \mathbf{r}} \times \mathbf{H}-\frac{\partial \mathbf{D}}{\partial t}\right\}
\end{aligned}
$$

or

$$
\begin{aligned}
-\left[1+\frac{\tau \sigma}{\varepsilon_{0} \varepsilon}\right] \frac{\partial^{2}}{\partial t^{2}} \frac{\partial \mathbf{B}}{\partial t}= & v_{\phi}^{2} \frac{\partial}{\partial \mathbf{r}} \times\left\{\Delta \mathbf{E}-\frac{\partial}{\partial \mathbf{r}}\left[\frac{\partial}{\partial \mathbf{r}} \cdot \mathbf{E}\right]\right\}+\frac{\sigma}{\varepsilon_{0} \varepsilon} \frac{\partial}{\partial t} \frac{\partial \mathbf{B}}{\partial t} \\
& +\frac{\tau}{\tau_{r}} \frac{1}{\varepsilon \varepsilon_{0}} \frac{\partial}{\partial t} \frac{\partial}{\partial \mathbf{r}} \times\left[\frac{\partial}{\partial \mathbf{r}} \times \mathbf{H}\right]-\frac{\tau}{\tau_{r}} \frac{1}{\varepsilon \varepsilon_{0}} \frac{\partial^{2}}{\partial t^{2}} \frac{\partial}{\partial \mathbf{r}} \times \mathbf{D}
\end{aligned}
$$


or

$$
\begin{aligned}
-\left[1+\frac{\tau \sigma}{\varepsilon_{0} \varepsilon}\right] \frac{\partial^{2}}{\partial t^{2}} \frac{\partial \mathbf{B}}{\partial t}= & v_{\phi}^{2} \frac{\partial}{\partial \mathbf{r}} \times\left\{\Delta \mathbf{E}-\frac{\partial}{\partial \mathbf{r}}\left[\frac{\partial}{\partial \mathbf{r}} \cdot \mathbf{E}\right]\right\}+\frac{\sigma}{\varepsilon_{0} \varepsilon} \frac{\partial}{\partial t} \frac{\partial \mathbf{B}}{\partial t} \\
& +\frac{\tau}{\tau_{r}} \frac{1}{\varepsilon_{0} \varepsilon} \frac{\partial}{\partial t} \frac{\partial}{\partial \mathbf{r}} \times\left[\frac{\partial}{\partial \mathbf{r}} \times \mathbf{H}\right]+\frac{\tau}{\tau_{r}} \frac{\partial}{\partial t} \frac{\partial^{2} \mathbf{B}}{\partial t^{2}}
\end{aligned}
$$

Let us use the identity

$$
\frac{\partial}{\partial \mathbf{r}} \times\left(\frac{\partial}{\partial \mathbf{r}} \times \mathbf{B}\right) \equiv \frac{\partial}{\partial \mathbf{r}}\left(\frac{\partial}{\partial \mathbf{r}} \cdot \mathbf{B}\right)-\Delta \mathbf{B}
$$

and the Maxwell relation

$$
\frac{\partial}{\partial \mathbf{r}} \cdot \mathbf{B}=0
$$

we have

$$
\begin{aligned}
-\left[1+\frac{\tau \sigma}{\varepsilon_{0} \varepsilon}\right] \frac{\partial^{2}}{\partial t^{2}} \frac{\partial \mathbf{B}}{\partial t}= & v_{\phi}^{2} \frac{\partial}{\partial \mathbf{r}} \times\left\{\Delta \mathbf{E}-\frac{\partial}{\partial \mathbf{r}}\left[\frac{\partial}{\partial \mathbf{r}} \cdot \mathbf{E}\right]\right\}+\frac{\sigma}{\varepsilon_{0} \varepsilon} \frac{\partial}{\partial t} \frac{\partial \mathbf{B}}{\partial t} \\
& -\frac{\tau}{\tau_{r}} \frac{1}{\mu_{0} \mu} \frac{1}{\varepsilon_{0} \varepsilon} \frac{\partial}{\partial t} \Delta \mathbf{B}+\frac{\tau}{\tau_{r}} \frac{\partial}{\partial t} \frac{\partial^{2} \mathbf{B}}{\partial t^{2}}
\end{aligned}
$$

or, if the reverse relaxation time is

$$
\frac{1}{\tau_{r}}=\frac{\sigma}{\varepsilon_{0} \varepsilon}
$$

we have

$$
\left[1+2 \frac{\tau}{\tau_{r}}\right] \frac{\partial^{2}}{\partial t^{2}} \frac{\partial \mathbf{B}}{\partial t}+\frac{1}{\tau_{r}} \frac{\partial}{\partial t} \frac{\partial \mathbf{B}}{\partial t}=\frac{\tau}{\tau_{r}} v_{\phi}^{2} \frac{\partial}{\partial t} \Delta \mathbf{B}-v_{\phi}^{2} \frac{\partial}{\partial \mathbf{r}} \times\left\{\Delta \mathbf{E}-\frac{\partial}{\partial \mathbf{r}}\left[\frac{\partial}{\partial \mathbf{r}} \cdot \mathbf{E}\right]\right\} \cdot
$$

Then

$$
\left[1+2 \frac{\tau}{\tau_{r}}\right] \frac{\partial^{2}}{\partial t^{2}} \frac{\partial \mathbf{B}}{\partial t}+\frac{1}{\tau_{r}} \frac{\partial}{\partial t} \frac{\partial \mathbf{B}}{\partial t}=\frac{\tau}{\tau_{r}} v_{\phi}^{2} \frac{\partial}{\partial t} \Delta \mathbf{B}+v_{\phi}^{2} \frac{\partial}{\partial \mathbf{r}} \times\left\{\frac{\partial}{\partial \mathbf{r}} \times\left(\frac{\partial}{\partial \mathbf{r}} \times \mathbf{E}\right)\right\}
$$

or

$$
\left[1+2 \frac{\tau}{\tau_{r}}\right] \frac{\partial^{2}}{\partial t^{2}} \frac{\partial \mathbf{B}}{\partial t}+\frac{1}{\tau_{r}} \frac{\partial}{\partial t} \frac{\partial \mathbf{B}}{\partial t}=\frac{\tau}{\tau_{r}} v_{\phi}^{2} \frac{\partial}{\partial t} \Delta \mathbf{B}-v_{\phi}^{2} \frac{\partial}{\partial t}\left\{\frac{\partial}{\partial \mathbf{r}} \times\left[\frac{\partial}{\partial \mathbf{r}} \times \mathbf{B}\right]\right\}
$$

or after integration on time we reach

$$
\left[1+2 \frac{\tau}{\tau_{r}}\right] \frac{\partial^{2} \mathbf{B}}{\partial t^{2}}+\frac{1}{\tau_{r}} \frac{\partial \mathbf{B}}{\partial t}=\frac{\tau}{\tau_{r}} v_{\phi}^{2} \Delta \mathbf{B}-v_{\phi}^{2}\left\{\frac{\partial}{\partial \mathbf{r}} \times\left[\frac{\partial}{\partial \mathbf{r}} \times \mathbf{B}\right]\right\}+f(x, y, z) .
$$

\section{Quantization in the Theory of the Magnetic Field Penetration. Comparison of the Non-Local and London's Theories}

Let us consider the $1 \mathrm{D}$ non-stationary electron evolution in plasma. We suppose that

$$
\tau \ll \tau_{r}
$$


After using (11.1) we have from (10.23)

$$
\frac{\partial^{2} B}{\partial t^{2}}+\frac{1}{\mathrm{~T}_{r}} \frac{\partial B}{\partial t}=v_{\phi}^{2} \frac{\partial^{2} B}{\partial x^{2}}+f(x) .
$$

In the following $\mathrm{T}_{r}$ and $\mathrm{L}_{r}$ are character time and length relaxation. Let us remind the London's equation written here as

$$
\Delta \mathbf{B}=\mu \mu_{0} \frac{n_{e} e^{2}}{m_{e}} \mathbf{B}
$$

or

$$
\Delta \mathbf{B}=\frac{1}{\ell^{2}} \mathbf{B},
$$

where $\ell$ is the London penetration depth.

$$
\ell=e \sqrt{\mu \mu_{0} \frac{n_{e}}{m_{e}}} .
$$

This London equation predicts that the magnetic field in a superconductor decays exponentially decays from whatever value it possesses at the surface.

We choose the function $f(x)$ in the form

$$
f(x) \rightarrow-\frac{v_{\phi}^{2}}{\mathrm{~L}_{r}^{2}} B,
$$

or

$$
f(x) \rightarrow-\frac{1}{\mathrm{~T}_{r}^{2}} B
$$

Relation

$$
\frac{\partial^{2} B}{\partial t^{2}}+\frac{1}{\mathrm{~T}_{r}} \frac{\partial B}{\partial t}=v_{\phi}^{2} \frac{\partial^{2} B}{\partial x^{2}}-\frac{1}{\mathrm{~T}_{r}^{2}} B_{0}(x)
$$

is telegraph equation which contains now $\tau_{r}=\mathrm{T}_{r}=$ const and $v_{\phi}=$ const as calculations parameters.

Solution of Equation (11.8) can be written in the form

$$
B(x, t)=\mathrm{e}^{-\frac{t}{2 \mathrm{~T}_{r}}} \sum_{n=1}^{\infty} B_{n} \sin \left(\lambda_{n} t\right) \cos \frac{\pi n x}{\mathrm{~L}_{r}}+B(0,0) \mathrm{e}^{-\frac{x}{\mathrm{~L}_{r}}},
$$

where $\mathrm{L}_{r}$ is the character scale of the relaxation length.

Solution (11.9) responds to natural initial and boundary conditions, namely

$$
\begin{gathered}
B(0, t)=\mathrm{e}^{-\frac{1}{2} \frac{t}{\mathrm{~T}_{r}}} \sum_{n=1}^{\infty} B_{n} \sin \left(\lambda_{n} t\right)+B(0,0), \\
B(0,0)=\text { const, } \\
B(x, t \rightarrow \infty)=B(0,0) \mathrm{e}^{-\frac{x}{\mathrm{~L}_{r}}}, \\
B(x \rightarrow \infty, t)=\mathrm{e}^{-\frac{1}{2} \frac{t}{\mathrm{~T}_{r}}} \sum_{n=1}^{\infty} B_{n} \sin \left(\lambda_{n} t\right) \cos \frac{\pi n x}{\mathrm{~L}_{r}} .
\end{gathered}
$$


Solution (11.9) can satisfy the Equation (11.8) if a condition should be satisfied. This condition can be found after the direct substitution of (11.9) in (11.8). We have

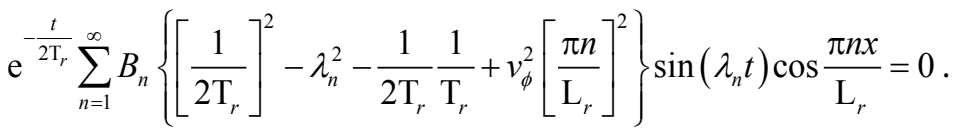

The relation (11.14) is fulfilled if the expression in the curly bracket is equal to zero.

$$
\left[\frac{1}{2 \mathrm{~T}_{r}}\right]^{2}-\lambda_{n}^{2}-\frac{1}{2 \mathrm{~T}_{r}} \frac{1}{\mathrm{~T}_{r}}+v_{\phi}^{2}\left[\frac{\pi n}{\mathrm{~L}_{r}}\right]^{2}=0
$$

or

$$
\lambda_{n}^{2} \mathrm{~T}_{r}^{2}=(\pi n)^{2}-\frac{1}{4}
$$

or

$$
\lambda_{n}=\frac{1}{\mathrm{~T}_{r}} \sqrt{(\pi n)^{2}-\frac{1}{4}} .
$$

As we see in the stationary case we have known equation

$$
v_{\phi}^{2} \frac{\partial^{2} B_{0}}{\partial x^{2}}=\frac{1}{\mathrm{~T}_{r}^{2}} B_{0},
$$

or

$$
\mathrm{L}_{r}^{2} \frac{\partial^{2} B_{0}}{\partial x^{2}}=B_{0}
$$

which has a solution

$$
B_{0}=B(0,0) \mathrm{e}^{-\frac{x}{\mathrm{~L}_{r}}}
$$

Finally, we have non-stationary solution as a wave with the time attenuation.

$$
\begin{gathered}
B(x, t)=\mathrm{e}^{-\frac{t}{2 \mathrm{~T}_{r}}} \sum_{n=1}^{\infty} B_{n} \sin \left(\lambda_{n} t\right) \cos \frac{\pi n x}{\mathrm{~L}_{r}}+B(0,0) \mathrm{e}^{-\frac{x}{\mathrm{~L}_{r}}} \\
\lambda_{n}=\frac{1}{\mathrm{~T}_{r}} \sqrt{(\pi n)^{2}-\frac{1}{4}} \sim \frac{1}{\mathrm{~T}_{r}} \pi n, n=1,2, \cdots
\end{gathered}
$$

and approximately we reach

$$
B(x, t)=\mathrm{e}^{-\frac{t}{2 \mathrm{~T}_{r}}} \sum_{n=1}^{\infty} B_{n} \sin \frac{\pi n t}{\mathrm{~T}_{r}}\left(\lambda_{n} t\right) \cos \frac{\pi n x}{\mathrm{~L}_{r}}+B(0,0) \mathrm{e}^{-\frac{x}{\mathrm{~L}_{r}}} .
$$

Some conclusions:

1) Relation (11.16) defines penetration of the longitudinal magnetic field in space.

2) This non-stationary attenuation has the character of wave damping.

3) The stationary case corresponds to the London's regime.

4) It is interesting to notice that the regime of the non-stationary wave attenu- 
ation exists for the evolution of the spherical object without fields (see Appendix 3).

\section{Nonlocal Pointing-Umov Theorem}

Let us obtain now the generalized nonlocal formulation of the Pointing-Umov theorem. In other words we intend to obtain the law of the energy conservation for electro-magnetic processes in the frame of non-local physics. We use the Equation (8.19) for following transformations.

$$
\begin{aligned}
{\left[1+\frac{\tau \sigma}{\varepsilon_{0} \varepsilon}\right] \frac{\partial^{2} \mathbf{E}}{\partial t^{2}}=} & v_{\phi}^{2}\left\{\Delta \mathbf{E}-\frac{\partial}{\partial \mathbf{r}}\left[\frac{\partial}{\partial \mathbf{r}} \cdot \mathbf{E}\right]\right\}-\frac{\sigma}{\varepsilon_{0} \varepsilon} \frac{\partial \mathbf{E}}{\partial t}-\frac{\tau}{\varepsilon_{0} \varepsilon} \frac{\partial}{\partial t}\left[\frac{\partial}{\partial \mathbf{r}} \cdot\left(\sigma \mathbf{E v}_{0}\right)\right] \\
& -\frac{\tau}{\varepsilon_{0} \varepsilon} \frac{\partial}{\partial t}\left[\frac{q}{m} \frac{\partial}{\partial \mathbf{r}}\left(n k_{B} T\right)-n q \mathbf{g}\right]
\end{aligned}
$$

We use the vector identity

$$
\frac{\partial}{\partial \mathbf{r}} \times\left(\frac{\partial}{\partial \mathbf{r}} \times \mathbf{E}\right) \equiv \frac{\partial}{\partial \mathbf{r}}\left(\frac{\partial}{\partial \mathbf{r}} \cdot \mathbf{E}\right)-\Delta \mathbf{E},
$$

then

$$
\begin{aligned}
{\left[1+\frac{\tau \sigma}{\varepsilon_{0} \varepsilon}\right] \frac{\partial^{2} \mathbf{E}}{\partial t^{2}}=} & -v_{\phi}^{2}\left\{\frac{\partial}{\partial \mathbf{r}} \times\left(\frac{\partial}{\partial \mathbf{r}} \times \mathbf{E}\right)\right\}-\frac{\sigma}{\varepsilon_{0} \varepsilon} \frac{\partial \mathbf{E}}{\partial t}-\frac{\tau}{\varepsilon_{0} \varepsilon} \frac{\partial}{\partial t}\left[\frac{\partial}{\partial \mathbf{r}} \cdot\left(\sigma \mathbf{E v}_{0}\right)\right] \\
& -\frac{\tau}{\varepsilon_{0} \varepsilon} \frac{\partial}{\partial t}\left[\frac{q}{m} \frac{\partial}{\partial \mathbf{r}}\left(n k_{B} T\right)-n q \mathbf{g}\right] .
\end{aligned}
$$

Let us transform now the first term of the right-hand-side of (12.3)

$$
\begin{aligned}
{\left[1+\frac{\tau \sigma}{\varepsilon_{0} \varepsilon}\right] \frac{\partial^{2} \mathbf{E}}{\partial t^{2}}=} & v_{\phi}^{2} \frac{\partial}{\partial t}\left\{\frac{\partial}{\partial \mathbf{r}} \times \mathbf{B}\right\}-\frac{\sigma}{\varepsilon_{0} \varepsilon} \frac{\partial \mathbf{E}}{\partial t}-\frac{\tau}{\varepsilon_{0} \varepsilon} \frac{\partial}{\partial t}\left[\frac{\partial}{\partial \mathbf{r}} \cdot\left(\sigma \mathbf{E v}_{0}\right)\right] \\
& -\frac{\tau}{\varepsilon_{0} \varepsilon} \frac{\partial}{\partial t}\left[\frac{q}{m} \frac{\partial}{\partial \mathbf{r}}\left(n k_{B} T\right)-n q \mathbf{g}\right] .
\end{aligned}
$$

After integration on time, we reach

$$
\begin{aligned}
{\left[1+\frac{\tau \sigma}{\varepsilon_{0} \varepsilon}\right] \frac{\partial \mathbf{E}}{\partial t}=} & v_{\phi}^{2}\left\{\frac{\partial}{\partial \mathbf{r}} \times \mathbf{B}\right\}-\frac{\sigma}{\varepsilon_{0} \varepsilon} \mathbf{E}-\frac{\tau}{\varepsilon_{0} \varepsilon}\left[\frac{\partial}{\partial \mathbf{r}} \cdot\left(\sigma \mathbf{E v}_{0}\right)\right] \\
& -\frac{\tau}{\varepsilon_{0} \varepsilon}\left[\frac{q}{m} \frac{\partial}{\partial \mathbf{r}}\left(n k_{B} T\right)-n q \mathbf{g}\right]+\mathbf{F}(x, y, z),
\end{aligned}
$$

where a function $\mathbf{F}(x, y, z)$ is defined by the initial and boundary conditions. Scalar multiplication by $\mathbf{E}$ of the both parts of Equation (12.5) gives the relation

$$
\begin{aligned}
{\left[1+\frac{\tau \sigma}{\varepsilon_{0} \varepsilon}\right] \mathbf{E} \cdot \frac{\partial \mathbf{E}}{\partial t}=} & v_{\phi}^{2} \mathbf{E} \cdot\left\{\frac{\partial}{\partial \mathbf{r}} \times \mathbf{B}\right\}-\frac{\sigma}{\varepsilon_{0} \varepsilon} E^{2}-\frac{\tau}{\varepsilon_{0} \varepsilon} \mathbf{E} \cdot\left[\frac{\partial}{\partial \mathbf{r}} \cdot\left(\sigma \mathbf{E v}_{0}\right)\right] \\
& -\frac{\tau}{\varepsilon_{0} \varepsilon} \mathbf{E} \cdot\left[\frac{q}{m} \frac{\partial}{\partial \mathbf{r}}\left(n k_{B} T\right)-n q \mathbf{g}\right]+\mathbf{E} \cdot \mathbf{F}(x, y, z) .
\end{aligned}
$$

Let us use the vector identity

$$
\mathbf{E} \cdot\left\{\frac{\partial}{\partial \mathbf{r}} \times \mathbf{B}\right\} \equiv \mu_{0} \mu \mathbf{H} \cdot\left\{\frac{\partial}{\partial \mathbf{r}} \times \mathbf{E}\right\}-\mu_{0} \mu \frac{\partial}{\partial \mathbf{r}} \cdot[\mathbf{E} \times \mathbf{H}] .
$$


We find

$$
\begin{aligned}
\frac{1}{2}\left[1+\frac{\tau \sigma}{\varepsilon_{0} \varepsilon}\right] \frac{\partial E^{2}}{\partial t}= & v_{\phi}^{2}\left\{\mu_{0} \mu \mathbf{H} \cdot\left\{\frac{\partial}{\partial \mathbf{r}} \times \mathbf{E}\right\}-\mu_{0} \mu \frac{\partial}{\partial \mathbf{r}} \cdot[\mathbf{E} \times \mathbf{H}]\right\} \\
& -\frac{\sigma}{\varepsilon_{0} \varepsilon} E^{2}-\frac{\tau}{\varepsilon_{0} \varepsilon} \mathbf{E} \cdot\left[\frac{\partial}{\partial \mathbf{r}} \cdot\left(\sigma \mathbf{E v}_{0}\right)\right] \\
& -\frac{\tau}{\varepsilon_{0} \varepsilon} \mathbf{E} \cdot\left[\frac{q}{m} \frac{\partial}{\partial \mathbf{r}}\left(n k_{B} T\right)-n q \mathbf{g}\right]+\mathbf{E} \cdot \mathbf{F}(x, y, z)
\end{aligned}
$$

or (for simplicity $\sigma=$ const,$\varepsilon=$ const and $\mu=$ const)

$$
\begin{aligned}
{\left[1+\frac{\tau \sigma}{\varepsilon_{0} \varepsilon}\right] \frac{\partial}{\partial t} \frac{\varepsilon_{0} \varepsilon E^{2}}{2} } & =\mathbf{H} \cdot\left\{\frac{\partial}{\partial \mathbf{r}} \times \mathbf{E}\right\}-\frac{\partial}{\partial \mathbf{r}} \cdot[\mathbf{E} \times \mathbf{H}]-\sigma E^{2}-\tau \mathbf{E} \cdot\left[\frac{\partial}{\partial \mathbf{r}} \cdot\left(\sigma \mathbf{E v}_{0}\right)\right] \\
& -\tau \mathbf{E} \cdot\left[\frac{q}{m} \frac{\partial}{\partial \mathbf{r}}\left(n k_{B} T\right)-n q \mathbf{g}\right]+\varepsilon_{0} \varepsilon \mathbf{E} \cdot \mathbf{F}(x, y, z)
\end{aligned}
$$

or

$$
\begin{aligned}
{\left[1+\frac{\tau \sigma}{\varepsilon_{0} \varepsilon}\right] \frac{\partial}{\partial t} \frac{\varepsilon_{0} \varepsilon E^{2}}{2} } & =-\mathbf{H} \cdot \frac{\partial \mathbf{B}}{\partial t}-\frac{\partial}{\partial \mathbf{r}} \cdot[\mathbf{E} \times \mathbf{H}]-\sigma E^{2} \\
& -\tau \mathbf{E} \cdot\left[\frac{\partial}{\partial \mathbf{r}} \cdot\left(\sigma \mathbf{E v}_{0}\right)+\frac{q}{m} \frac{\partial p}{\partial \mathbf{r}}-n q \mathbf{g}\right]+\varepsilon_{0} \varepsilon \mathbf{E} \cdot \mathbf{F}(x, y, z)
\end{aligned}
$$

or

$$
\begin{aligned}
& \frac{\partial}{\partial t} \frac{\varepsilon_{0} \varepsilon E^{2}+\mu_{0} \mu H^{2}}{2}+\frac{\tau \sigma}{\varepsilon_{0} \varepsilon} \frac{\partial}{\partial t} \frac{\varepsilon_{0} \varepsilon E^{2}}{2} \\
& =-\frac{\partial}{\partial \mathbf{r}} \cdot[\mathbf{E} \times \mathbf{H}]-\sigma E^{2}-\tau \mathbf{E} \cdot\left[\frac{\partial}{\partial \mathbf{r}} \cdot\left(\sigma \mathbf{E v}_{0}\right)+\frac{q}{m} \frac{\partial}{\partial \mathbf{r}}\left(n k_{B} T\right)-n q \mathbf{g}\right] \\
& +\varepsilon_{0} \varepsilon \mathbf{E} \cdot \mathbf{F}(x, y, z) .
\end{aligned}
$$

It is the nonlocal formulation of the Umov-Pointing theorem which takes into account transport processes in physical system. In the local case we find

$$
\frac{\partial}{\partial t} \frac{\varepsilon_{0} \varepsilon E^{2}+\mu_{0} \mu H^{2}}{2}=-\frac{\partial}{\partial \mathbf{r}} \cdot[\mathbf{E} \times \mathbf{H}]-\sigma E^{2}+\varepsilon_{0} \varepsilon \mathbf{E} \cdot \mathbf{F}(x, y, z) .
$$

As usual a function $\mathbf{F}(x, y, z)$ is originated by integration on time and can be taken as zero; we obtain the classical formulation of the Umov-Pointing theorem.

$$
\sigma E^{2}=-\frac{\partial}{\partial t}\left[\frac{\varepsilon_{0} \varepsilon E^{2}+\mu_{0} \mu H^{2}}{2}\right]-\operatorname{div}[\mathbf{E}, \mathbf{H}] .
$$

This equation is well known local energy equation, where the left hand side of this equation corresponds to the Joule heating.

\section{The Soliton Movement in One Species Physical System about the Heat Transfer in Graphene}

Particular attention of researchers has been recently attracted to a new carbon material, i.e., graphene, consisting of a single layer of carbon atoms and having a 
planar hexagonal structure. Electromagnetic waves propagating in carbon structures become highly nonlinear even at relatively weak fields, which results in possible propagation of electromagnetic solitary waves (which are soliton analogs, or even solitons) in carbon nanotubes and graphene (see review in [31] [32] [33]). The discussed properties of carbon structures have generated both increased theoretical interest and attempts at application in nonlinear optical devices.

The fundamental result also consists in discovering of the extremely high thermal conductivity in two-dimensional crystals including graphene. This effect (which takes place even in room-temperature) can be explained as a result of soliton movements without destruction. This effect was forecasted by me many years ago. Really, the transport processes in graphene as the effect of the soliton movement are investigated by me many years ago. The fundamental monograph [5] contains Chapter 6 (Quantum Solitons in Solid Matter), Item 6.2 (Application of non-local quantum hydrodynamics to the description of the charge density waves in the graphene crystal lattice) with the fundamental conclusion (p. 178): "Important conclusion: high temperature superconductors demonstrate new type of electronic order and modulation of atomic positions. The above mentioned graphene properties can be explained only in the frame of the self-consistent non-local quantum theory which leads to the appearance of the soliton waves moving in graphene."

Then it is impossible to talk about individual particles as heating transfer carriers. In the definite sense the high temperature super conductivity and super heat conductivity have the same origin-the appearance of moving solitons without destruction.

Let us demonstrate the example of the electron soliton movement in 1D physical system.

We transform nonlocal Maxwell equation

$$
\frac{\partial}{\partial x} E=4 \pi\left[\rho_{e}-\tau\left(\frac{\partial \rho_{e}}{\partial t}-\frac{\partial}{\partial x}\left(\rho_{e} u\right)\right)\right],
$$

where $\rho_{e}$ is the electron charge density, or

$$
-\frac{\partial^{2}}{\partial x^{2}} U_{e}=4 \pi\left[n_{e}-\tau\left(\frac{\partial n_{e}}{\partial t}-\frac{\partial}{\partial x}\left(n_{e} u\right)\right)\right] e,
$$

where $U_{e}$ is electrical potential and $n_{e}$ is the electron number density. Equation (13.2) can be written in terms of wave parameters $(\xi=x-C t)$. Namely

$$
-\frac{\partial^{2}}{\partial \xi^{2}} U_{e}=4 \pi \frac{e}{m_{e}}\left[\rho-\tau\left(-C \frac{\partial \rho}{\partial \xi}-\frac{\partial}{\partial \xi}(\rho u)\right)\right],
$$

where $\rho$ is the mass density, or introducing the absolute electron charge and potential $U=U_{e} / m_{e}$ we find

$$
\frac{\partial^{2}}{\partial \xi^{2}} U=4 \pi \frac{|e|}{m_{e}^{2}}\left[\rho+\tau\left(C \frac{\partial \rho}{\partial \xi}+\frac{\partial}{\partial \xi}(\rho u)\right)\right] .
$$


We use the following system of scales:

$$
\begin{gathered}
C_{0}=x_{0} \frac{1}{\tau_{0}}, \tilde{C}=1, \rho_{0}=\frac{m_{e}}{4 \pi x_{0}^{3}}, U_{0}=\frac{|e|}{m_{e} x_{0}}, u_{0}=\sqrt{\frac{\hbar}{4 m_{e}}}=\frac{1}{2} \sqrt{\frac{\hbar}{m_{e}}}, \\
\tau=\tau^{(q u)}=t_{0} \frac{1}{\tilde{u}^{2}}=t_{0} \frac{u_{0}^{2}}{u^{2}}=\frac{\hbar}{4 m_{e}} \frac{1}{u^{2}} \tau_{0}, \tau_{0}=\frac{x_{0}}{u_{0}}=2 x_{0} \sqrt{\frac{m_{e}}{\hbar}} \\
C_{0}=2 \sqrt{\frac{\hbar}{m_{e}}}=2.1519 \frac{\mathrm{cm}}{\mathrm{s}} .
\end{gathered}
$$

The single independent scale is $x_{0}$.

Taking into account $\hbar=1.054572 \times 10^{-27} \mathrm{erg} \cdot \mathrm{s}, m_{e}=0.9109383 \times 10^{-27} \mathrm{~g}$, we have

$$
\sqrt{\frac{\hbar}{m_{e}}}=1.0759538 \frac{\mathrm{cm}}{\mathrm{s}}, \sqrt{\frac{m_{e}}{\hbar}}=0.964058 \frac{\mathrm{s}}{\mathrm{cm}}, \quad \tilde{\tau}=\frac{1}{\tilde{u}^{2}} .
$$

The considered physical system works in Meissner regime, and then we needn't to use the influence of magnetic field. We reach the system of dimensionless equations

$$
\begin{aligned}
& \frac{\partial^{2}}{\partial \tilde{\xi}^{2}} \tilde{U}=\tilde{\rho}+\frac{1}{\tilde{u}^{2}} \frac{\partial}{\partial \tilde{\xi}}[\tilde{\rho}(1+\tilde{u})] \\
& \frac{\partial \tilde{\rho}}{\partial \tilde{\xi}}-\frac{\partial \tilde{\rho} \tilde{u}}{\partial \tilde{\xi}}+\frac{\partial}{\partial \tilde{\xi}}\left\{\frac{1}{\tilde{u}^{2}}\left[\frac{\partial}{\partial \tilde{\xi}}\left(\tilde{p}+\tilde{\rho}+\tilde{\rho} \tilde{u}^{2}-2 \tilde{\rho} \tilde{u}\right)+\tilde{\rho} \frac{\partial \tilde{U}}{\partial \tilde{\xi}}\right]\right\}=0 \\
& \frac{\partial}{\partial \tilde{\xi}}\left(\tilde{\rho} \tilde{u}^{2}+\tilde{p}-\tilde{\rho} \tilde{u}\right)+\frac{\partial}{\partial \tilde{\xi}}\left\{\frac{1}{\tilde{u}^{2}}\left[\frac{\partial}{\partial \tilde{\xi}}\left(2 \tilde{\rho} \tilde{u}^{2}-\tilde{\rho} \tilde{u}+2 \tilde{p}-\tilde{\rho} \tilde{u}^{3}-3 \tilde{p} \tilde{u}\right)+\tilde{\rho} \frac{\partial \tilde{U}}{\partial \tilde{\xi}}\right]\right\} \\
& +\frac{\partial \tilde{U}}{\partial \tilde{\xi}}\left\{\tilde{\rho}-\frac{1}{\tilde{u}^{2}}\left[-\frac{\partial \tilde{\rho}}{\partial \tilde{\xi}}+\frac{\partial}{\partial \tilde{\xi}}(\tilde{\rho} \tilde{u})\right]\right\}-2 \frac{\partial}{\partial \tilde{\xi}}\left\{\frac{\tilde{\rho}}{\tilde{u}} \frac{\partial \tilde{U}}{\partial \tilde{\xi}}\right\}=0 \\
& \quad \frac{\partial}{\partial \tilde{\xi}}\left(\tilde{\rho} \tilde{u}^{2}+3 \tilde{p}-\tilde{\rho} \tilde{u}^{3}-5 \tilde{p} \tilde{u}\right) \\
& \quad-\frac{\partial}{\partial \tilde{\xi}}\left\{\frac{1}{\tilde{u}^{2}} \frac{\partial}{\partial \tilde{\xi}}\left(2 \tilde{\rho} \tilde{u}^{3}+10 \tilde{p} \tilde{u}-\tilde{\rho} \tilde{u}^{2}-3 \tilde{p}-\tilde{\rho} \tilde{u}^{4}-8 \tilde{p} \tilde{u}^{2}-5 \frac{\tilde{p}^{2}}{\tilde{\rho}}\right)\right\} \\
& +\frac{\partial}{\partial \tilde{\xi}}\left\{\frac{1}{\tilde{u}^{2}}\left(3 \tilde{\rho} \tilde{u}^{2}+5 \tilde{p}\right) \frac{\partial \tilde{U}}{\partial \tilde{\xi}}\right\}-2 \tilde{\rho} \tilde{u} \frac{\partial \tilde{U}}{\partial \tilde{\xi}}-2 \frac{\partial}{\partial \tilde{\xi}}\left\{\frac{\tilde{\rho}}{\tilde{u}} \frac{\partial \tilde{U}}{\partial \tilde{\xi}}\right\} \\
& \quad+\frac{2}{\tilde{u}^{2}} \frac{\partial \tilde{U}}{\partial \tilde{\xi}}\left[-\frac{\partial}{\partial \tilde{\xi}}(\tilde{\rho} \tilde{u})+\frac{\partial}{\partial \tilde{\xi}}\left(\tilde{\rho} \tilde{u}^{2}+\tilde{p}\right)+\tilde{\rho} \frac{\partial \tilde{U}}{\partial \tilde{\xi}}\right]=0,
\end{aligned}
$$

Appendix 2 contains the corresponding Maple program.

The following Figure 13 reflects the result of calculations for the Cauchy conditions:

$\mathrm{u}(0)=1, \mathrm{p}(0)=2, \mathrm{r}(0)=1, \mathrm{D}(\mathrm{u})(0)=0, \mathrm{D}(\mathrm{p})(0)=0, \mathrm{D}(\mathrm{r})(0)=0, \mathrm{D}(\mathrm{v})(0)=0, \mathrm{v}(0)=1$.

As we see from Figure 13: the pressure distribution (leading to the heat transport) and the density distribution (leading to the charge transport) have the character of moving solitons. 
r-solid line, u-dashed line, p-dashdot line, v-dotted line

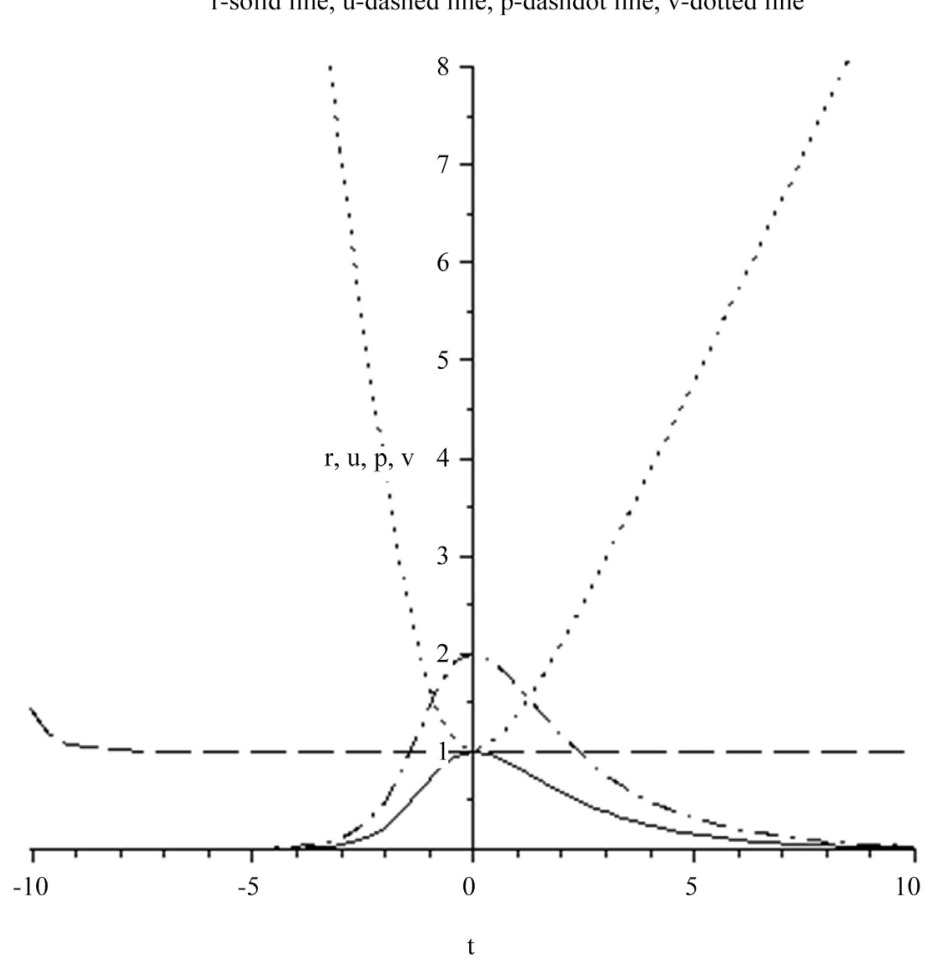

Figure 13. Density $\mathrm{r}(\tilde{\rho})$ —solid line, velocity u $(\tilde{u})$-dashed line, pressure $\mathrm{p}(\tilde{p})$-dash-dot line, self-consistent potential $\mathrm{v}(\tilde{U})$-dotted line.

\section{Discussion, Principal Derivations, Conclusion and Proposals}

Research of superconductors is curried out very actively. But in spite of obvious success, the following conclusion could be established:

1) Contemporary theories of superconductivity based on the Schrödinger equation, practically exhaust their arguments and have no possibility to explain effects of the high temperature superconductivity.

2) Contemporary theories of superconductivity (including BCS) based on the Schrödinger equation, can't propose the principles of search and creation of superconducting materials.

The most impressive demonstration of these difficulties consists in the fundamental distinction between a strange metal and a conventional metal; in other words in the absence of well-defined quasi-particles in the frame of local physics. This is manifested in transport properties which defy conventional theory, the most famous of which is a T-linear resistivity that persists from nearly $0 \mathrm{~K}$ to high temperatures above the proposed Mott-Ioe-Regel (MIR) limit, beyond which Boltzmann theory ceases to be valid.

Without exception, all existing before proposals fail this test. Most of these theories depart from the assumption that the electrical currents are carried by one or the other system of quasi-particles. This is fundamental: it is impossible to identify the simplicity principle dealing with particle physics. The transport is 
assumed to be due to thermally excited quasi-particles behaving like classical balls being scattered in various ways, dumping eventually their momentum in the lattice. However, it is a matter of principle that the physics of such quasi-particles in real solids is never simple. These interact with phonons which are very efficient sources of momentum dissipation which should be strongly temperature dependent for elementary reasons.

The conclusion is that quasi-particles are quantum solitons which are moving without destruction.

From position of the quantum non-local hydrodynamics, the problem of search and creation of superconductive materials come to the search of materials which lattices ensure the soliton movement without destruction. In my opinion, the mentioned materials can be created artificially using the technology of the special introduction of quantum dots in matrices on the basement of proposed quantum hydrodynamics. It is known that technology of material creation with special quantum dots exists now in other applications.

\section{Conflicts of Interest}

The author declares no conflicts of interest regarding the publication of this paper.

\section{References}

[1] Boltzmann, L. (1872) Weitere Studien über das Wärmegleichgewicht unter Gasmolekulen. Sitz. Ber. Kaiserl. Akad. Wiss. 66(2) s.275.

[2] Boltzmann, L. (1912) Vorlesungen über Gastheorie. Leipzig: Verlag von Johann Barth. Zweiter unveränderten Abdruck. 2 Teile.

[3] Alekseev, B.V. (1982) Matematicheskaya Kinetika Reagiruyushchikh Gazov (Mathematical Theory of Reacting Gases). Nauka, Moscow.

[4] Alexeev, B.V. (2004) Generalized Boltzmann Physical Kinetics. Elsevier, Amsterdam, 368 p. https://doi.org/10.1016/B978-044451582-7/50027-8

[5] Alexeev, B.V. (2015) Unified Non-Local Theory of Transport Processes. Elsevier, Amsterdam, $644 \mathrm{p}$.

[6] Alexeev, B.V. (2016) Unified Non-Local Relativistic Theory of Transport Processes. Elsevier, Amsterdam, 455 p.

[7] Alexeev, B.V. (2017) Nonlocal Astrophysics. Dark Matter, Dark Energy, Physical Vacuum. Elsevier, Amsterdam, 454 p.

[8] Alexeev, B.V. (1994) Philosophical Transactions of the Royal Society of London, 349, 417-443. https://doi.org/10.1098/rsta.1994.0140

[9] Alexeev, B.V. (1995) Physica A, 216, 459-468. https://doi.org/10.1016/0378-4371(95)00044-8

[10] Alekseev, B.V. (2000) Physics-Uspekhi, 170, 601-629. https://doi.org/10.1070/PU2000v043n06ABEH000694

[11] Alekseev, B.V. (2003) Physics-Uspekhi, 173, 139-167. https://doi.org/10.1070/PU2003v046n02ABEH001221

[12] Bogolyubov, N.N. (1946) Problemy Dinamicheskoi Teorii v Statisticheskoi Fizike (Dynamic Theory Problems in Statistical Physics). Gostekhizdat, Moscow Lenin- 
grad. [Translated into English The Dynamical Theory in Statistical Physics (Hindustan Publ. Corp., Delhi, 1965)]

[13] Born, M. and Green, H.S. (1946) Proceedings of the Royal Society, 188, 10. https://doi.org/10.1098/rspa.1946.0093

[14] Green, H.S. (1952) The Molecular Theory of Fluids. North-Holland Publishing Company, Amsterdam.

[15] Kirkwood, J.G. (1947) The Journal of Chemical Physics, 15, 72. https://doi.org/10.1063/1.1746292

[16] Yvon, J. (1935) La Theorie Statistique des Fluide et l'Equation d'etat. Hermann \& Cie, Paris.

[17] Bhatnagar, P.L., Gross, E.P. and Krook, M.A. (1954) Physical Review, 94, 511-525. https://doi.org/10.1103/PhysRev.94.511

[18] Alexeev, B.V. (2008) Journal of Nanoelectronics and Optoelectronics, 3, 143-158. https://doi.org/10.1166/jno.2008.207

[19] Alexeev, B.V. (2008) Journal of Nanoelectronics and Optoelectronics, 3, 316-328. https://doi.org/10.1166/jno.2008.311

[20] Ehrenfest, P. (1979) Collected Scientific Papers. North-Holland Publ. Co., Amsterdam.

[21] Madelung, E. (1927) Zeitschrift für Physik, 40, 322-326. https://doi.org/10.1007/BF01400372

[22] Bell, J.S. (1964) Physics, 1, 195-290. https://doi.org/10.1103/PhysicsPhysiqueFizika.1.195

[23] Homes, C.C., et al. (2004) Nature, 430, 539-541. https://doi.org/10.1038/nature02673

[24] Hartnoll, S.A. (2014) Nature Physics, 11, 54. https://doi.org/10.1038/nphys3174

[25] Zaanen, J. (2004) Nature, 430, 512-513. https://doi.org/10.1038/430512a

[26] Zaanen, J. (2018) SciPost Physics, 6, 61. https://doi.org/10.21468/SciPostPhys.6.5.061

[27] Krömer, H. (1958) Physical Review, 109, 1856. https://doi.org/10.1103/PhysRev.109.1856

[28] Ryzhii, V.I. (1969) Soviet Physics, Solid State, 11, 1995.

[29] Ryzhii, V.I. (2005) Uspekhi Fizicheskikh Nauk, 175, 205. https://doi.org/10.1070/PU2005v048n02ABEH002104

[30] Monstein, C. and Wesley, J.P. (2002) Europhysics Letters, 59, 514-520. https://doi.org/10.1209/epl/i2002-00136-9

[31] Novoselov, K.S., Geim, A.K., Morozov, S.V., Jiang, D., Zhang, Y., Dubonos, S.V., Grigorieva, I.V. and Firsov, A.A. (2004) Science, 306, 666. https://doi.org/10.1126/science.1102896

[32] Novoselov, K.S., Geim, A.K., Morozov, S.V., Jiang, D., Katsnelson, M.I., Grigorieva, I.V., Dubonos, S.V. and Firsov, A.A. (2005) Nature, 438, 197. https://doi.org/10.1038/nature04233

[33] Geim, A.K. and Novoselov, K.S. (2007) Nature Materials, 6, 183. https://doi.org/10.1038/nmat1849 


\section{Appendix 1}

Maple program for modeling of soliton motion in two species physical system.

(the program is ready for application)

The ratio $L / H$ is ratio of masses of the light and heavy particles; for example $L$ $=1, H=1838$.

dsolve[interactive] $(\{$

$\operatorname{diff}(v(t), t \$ 2)=-r(t)^{*}(\mathrm{~L} / \mathrm{H})+(\mathrm{L} / \mathrm{H})^{\wedge} 2^{*} \operatorname{diff}\left(\mathrm{r}(\mathrm{t})^{*} \mathrm{u}(\mathrm{t})-\mathrm{r}(\mathrm{t}), \mathrm{t}\right) / \mathrm{u}(\mathrm{t}) \wedge 2+$

$s(t)-\operatorname{diff}(s(t) * u(t)-s(t), t) / u(t)^{\wedge} 2$,

$\operatorname{diff}(r(t) *(1-u(t)), t)+(L / H) * \operatorname{diff}\left(\operatorname{diff}\left(p(t)+r(t)+r(t)^{*} u(t)^{\wedge} 2-\right.\right.$

$\left.\left.2^{\star} r(t)^{*} u(t), t\right) / u(t) \wedge 2, t\right)+(L / H)^{\wedge} 2^{*} \operatorname{diff}\left(r(t)^{*} \operatorname{diff}(v(t), t) / u(t) \wedge 2, t\right)=0$,

$\operatorname{diff}(s(t) *(1-u(t)), t)+\operatorname{diff}\left(\left(\operatorname{diff}\left(q(t)+s(t)+s(t)^{*} u(t) \wedge 2-2^{*} s(t)^{*} u(t), t\right)\right) / u(t) \wedge 2\right.$,

$\mathrm{t})-\operatorname{diff}\left(\mathrm{s}(\mathrm{t})^{*} \operatorname{diff}(\mathrm{v}(\mathrm{t}), \mathrm{t}) / \mathrm{u}(\mathrm{t})^{\wedge} 2, \mathrm{t}\right)=0$,

$\operatorname{diff}\left((\mathrm{r}(\mathrm{t})+\mathrm{s}(\mathrm{t}))^{*} \mathrm{u}(\mathrm{t}) \wedge 2+\mathrm{p}(\mathrm{t})+\mathrm{q}(\mathrm{t})-(\mathrm{r}(\mathrm{t})+\mathrm{s}(\mathrm{t}))^{*} \mathrm{u}(\mathrm{t}), \mathrm{t}\right)+$

$\operatorname{diff}\left(\operatorname{diff}\left(2^{*} r(t)^{*} u(t) \wedge 2+2^{*} p(t)-r(t) * u(t)-r(t)^{*} u(t) \wedge 3-\right.\right.$

$\left.\left.3^{*} \mathrm{p}(\mathrm{t})^{*} \mathrm{u}(\mathrm{t}), \mathrm{t}\right) /\left(\mathrm{u}(\mathrm{t}) \wedge 2^{*}(\mathrm{H} / \mathrm{L})\right), \mathrm{t}\right)+$

$\operatorname{diff}\left(\operatorname{diff}\left(2^{*} s(t)^{*} u(t) \wedge 2+2^{*} q(t)-s(t)^{*} u(t)-s(t)^{*} u(t)^{\wedge} 3-\right.\right.$

$\left.\left.3^{*} \mathrm{q}(\mathrm{t}) * \mathrm{u}(\mathrm{t}), \mathrm{t}\right) / \mathrm{u}(\mathrm{t})^{\wedge} 2, \mathrm{t}\right)+$

$\operatorname{diff}\left(\mathrm{r}(\mathrm{t}){ }^{*} \operatorname{diff}(\mathrm{v}(\mathrm{t}), \mathrm{t})^{*}(\mathrm{~L} / \mathrm{H})^{\wedge} 2 / \mathrm{u}(\mathrm{t}) \wedge 2, \mathrm{t}\right)-$

$\operatorname{diff}(s(t) * \operatorname{diff}(v(t), t) / u(t) \wedge 2, t)+$

$r(t)^{*} \operatorname{diff}(v(t), t)^{*}(\mathrm{~L} / \mathrm{H})-\mathrm{s}(\mathrm{t}) * \operatorname{diff}(\mathrm{v}(\mathrm{t}), \mathrm{t})-$

$(\mathrm{L} / \mathrm{H})^{\wedge} 2^{*} \operatorname{diff}(\mathrm{v}(\mathrm{t}), \mathrm{t})^{*} \operatorname{diff}\left(\mathrm{r}(\mathrm{t})^{*}(\mathrm{u}(\mathrm{t})-1), \mathrm{t}\right) / \mathrm{u}(\mathrm{t}) \wedge 2+$

$\operatorname{diff}(\mathrm{v}(\mathrm{t}), \mathrm{t})^{*} \operatorname{diff}\left(\mathrm{s}(\mathrm{t})^{*}(\mathrm{u}(\mathrm{t})-1), \mathrm{t}\right) / \mathrm{u}(\mathrm{t})^{\wedge} 2-$

$2^{*} \operatorname{diff}\left(\left((\mathrm{L} / \mathrm{H}) \wedge 2^{*} \mathrm{r}(\mathrm{t})-\mathrm{s}(\mathrm{t})\right)^{*} \operatorname{diff}(\mathrm{v}(\mathrm{t}), \mathrm{t}) / \mathrm{u}(\mathrm{t}), \mathrm{t}\right)=0$,

$\operatorname{diff}\left(\mathrm{r}(\mathrm{t})^{*} \mathrm{u}(\mathrm{t})^{\wedge} 3+5^{*} \mathrm{p}(\mathrm{t})^{*} \mathrm{u}(\mathrm{t})-\mathrm{r}(\mathrm{t})^{*} \mathrm{u}(\mathrm{t})^{\wedge} 2-3^{*} \mathrm{p}(\mathrm{t}), \mathrm{t}\right)+$

$(\mathrm{L} / \mathrm{H})^{*} \operatorname{diff}\left(\operatorname{diff}\left(2^{*} r(\mathrm{t})^{*} \mathrm{u}(\mathrm{t})^{\wedge} 3+10^{*} \mathrm{p}(\mathrm{t})^{*} \mathrm{u}(\mathrm{t})-\mathrm{r}(\mathrm{t})^{*} \mathrm{u}(\mathrm{t}) \wedge 4-8^{*} \mathrm{p}(\mathrm{t})^{*} \mathrm{u}(\mathrm{t})^{\wedge} 2-5^{*} \mathrm{p}(\right.\right.$

$\left.\left.\mathrm{t})^{\wedge} 2 / \mathrm{r}(\mathrm{t})-\mathrm{r}(\mathrm{t})^{*} \mathrm{u}(\mathrm{t})^{\wedge} 2-3^{*} \mathrm{p}(\mathrm{t}), \mathrm{t}\right) / \mathrm{u}(\mathrm{t})^{\wedge} 2, \mathrm{t}\right)+$

$(\mathrm{L} / \mathrm{H})^{\wedge} 2^{\star} \operatorname{diff}\left(\left(2^{*} \mathrm{r}(\mathrm{t})^{*} \mathrm{u}(\mathrm{t})-3^{*} \mathrm{r}(\mathrm{t})^{*} \mathrm{u}(\mathrm{t}) \wedge 2-5^{*} \mathrm{p}(\mathrm{t})\right)^{\star} \operatorname{diff}(\mathrm{v}(\mathrm{t}), \mathrm{t}) / \mathrm{u}(\mathrm{t})^{\wedge} 2, \mathrm{t}\right)+$

$2^{*}(\mathrm{~L} / \mathrm{H})^{*} \mathrm{r}(\mathrm{t}) * \operatorname{diff}(\mathrm{v}(\mathrm{t}), \mathrm{t})^{*} \mathrm{u}(\mathrm{t})-$

$2^{*}(\mathrm{~L} / \mathrm{H})^{\wedge} 2^{*} \operatorname{diff}(\mathrm{v}(\mathrm{t}), \mathrm{t})^{*} \operatorname{diff}\left(\mathrm{r}(\mathrm{t})^{*} \mathrm{u}(\mathrm{t}) \wedge 2+\mathrm{p}(\mathrm{t})-\mathrm{r}(\mathrm{t})^{*} \mathrm{u}(\mathrm{t}), \mathrm{t}\right) / \mathrm{u}(\mathrm{t})^{\wedge} 2-$

$2^{*}(\mathrm{~L} / \mathrm{H}) \wedge 3^{*} \mathrm{r}(\mathrm{t}) * \operatorname{diff}(\mathrm{v}(\mathrm{t}), \mathrm{t}) \wedge 2 / \mathrm{u}(\mathrm{t}) \wedge 2=-(\mathrm{p}(\mathrm{t})-\mathrm{q}(\mathrm{t}))^{*} \mathrm{u}(\mathrm{t}) \wedge 2^{*}((\mathrm{~L}+\mathrm{H}) / \mathrm{L})$,

$\operatorname{diff}\left(s(t)^{*} \mathrm{u}(\mathrm{t}) \wedge 3+5^{*} \mathrm{q}(\mathrm{t})^{*} \mathrm{u}(\mathrm{t})-\mathrm{s}(\mathrm{t})^{*} \mathrm{u}(\mathrm{t}) \wedge 2-3^{*} \mathrm{q}(\mathrm{t}), \mathrm{t}\right)+$

$\operatorname{diff}\left(\operatorname{diff}\left(2^{*} s(t)^{*} u(t) \wedge 3+10^{*} q(t)^{*} u(t)-s(t)^{*} u(t) \wedge 4-8^{*} q(t)^{*} u(t) \wedge 2-5^{*} q(t) \wedge 2 / s(\right.\right.$

t) $\left.\left.-s(t)^{*} u(t)^{\wedge} 2-3^{*} q(t), t\right) / u(t) \wedge 2, t\right)+$

$\operatorname{diff}\left(\operatorname{diff}(v(t), t)^{*}\left(3^{*} s(t)^{*} \mathrm{u}(\mathrm{t}) \wedge 2+5^{*} \mathrm{q}(\mathrm{t})-2^{*} \mathrm{~s}(\mathrm{t})^{*} \mathrm{u}(\mathrm{t})\right) / \mathrm{u}(\mathrm{t}) \wedge 2, \mathrm{t}\right)-$

$2^{*} s(t)^{*} \operatorname{diff}(v(t), t)^{*} u(t)+$

$2^{*} \operatorname{diff}(\mathrm{v}(\mathrm{t}), \mathrm{t})^{*} \operatorname{diff}\left(\mathrm{s}(\mathrm{t})^{*} \mathrm{u}(\mathrm{t})^{\wedge} 2+\mathrm{q}(\mathrm{t})-\mathrm{s}(\mathrm{t})^{*} \mathrm{u}(\mathrm{t}), \mathrm{t}\right) / \mathrm{u}(\mathrm{t}) \wedge 2-2^{*} \mathrm{~s}(\mathrm{t})^{*} \operatorname{diff}(\mathrm{v}(\mathrm{t}), \mathrm{t})^{\wedge} 2$

$/ \mathrm{u}(\mathrm{t}) \wedge 2=-(\mathrm{q}(\mathrm{t})-\mathrm{p}(\mathrm{t}))^{*} \mathrm{u}(\mathrm{t}) \wedge 2^{*}((\mathrm{~L}+\mathrm{H}) / \mathrm{L})$,

$\operatorname{diff}(\mathrm{R}(\mathrm{t}), \mathrm{t})=\mathrm{r}(\mathrm{t}), \operatorname{diff}(\mathrm{S}(\mathrm{t}), \mathrm{t})=\mathrm{s}(\mathrm{t})$

$\mathrm{v}(0)=1, \mathrm{r}(0)=1, \mathrm{~s}(0)=1 / 1838, \mathrm{u}(0)=1, \mathrm{p}(0)=1000, \mathrm{q}(0)=950, \mathrm{R}(0)=0, \mathrm{~S}(0)=0$,

$\mathrm{D}(\mathrm{v})(0)=0, \mathrm{D}(\mathrm{r})(0)=0, \mathrm{D}(\mathrm{s})(0)=0, \mathrm{D}(\mathrm{u})(0)=0, \mathrm{D}(\mathrm{p})(0)=0, \mathrm{D}(\mathrm{q})(0)=0$

\}$)$; 


\section{Appendix 2}

Maple program for modeling of soliton motion in one species physical system.

(the program is ready for application)

dsolve[interactive] $(\{$

$\operatorname{diff}\left(r(t)^{*}(1-u(t)), t\right)+\operatorname{diff}\left(\left(\operatorname{diff}\left(p(t)+r(t)+r(t)^{*} u(t)^{\wedge} 2-2^{*} r(t)^{*} u(t), t\right)\right) / u(t) \wedge 2\right.$, $t)+\operatorname{diff}(r(t) * \operatorname{diff}(v(t), t) / u(t) \wedge 2, t)=0$,

$\operatorname{diff}\left(r(t)^{*} u(t)^{\wedge} 2+p(t)-r(t)^{*} u(t), t\right)+\operatorname{diff}\left(\operatorname{diff}\left(2^{*} r(t)^{*} u(t) \wedge 2+2^{*} p(t)-r(t)^{*} u(t)-\right.\right.$ $\left.\left.r(t)^{*} u(t)^{\wedge} 3-3^{*} p(t)^{*} u(t), t\right) / u(t)^{\wedge} 2, t\right)+\operatorname{diff}\left(\operatorname{diff}(v(t), t)^{*} r(t) / u(t) \wedge 2, t\right)+r(t)^{*} \operatorname{diff}($ $\mathrm{v}(\mathrm{t}), \mathrm{t})-\operatorname{diff}(\mathrm{v}(\mathrm{t}), \mathrm{t})^{*} \operatorname{diff}\left(\mathrm{r}(\mathrm{t})^{*}(\mathrm{u}(\mathrm{t})-1), \mathrm{t}\right) / \mathrm{u}(\mathrm{t})^{\wedge} 2-$

$2^{*} \operatorname{diff}(\operatorname{diff}(v(t), t) * r(t) / u(t), t)=0$,

$\operatorname{diff}\left(r(t)^{*} u(t)^{\wedge} 2+3^{*} p(t)-r(t)^{*} u(t)^{\wedge} 3-5^{*} p(t)^{*} u(t), t\right)-\operatorname{diff}\left(\operatorname{diff}\left(2^{*} r(t)^{*} u(t)^{\wedge} 3+\right.\right.$ $\left.10^{*} \mathrm{p}(\mathrm{t})^{*} \mathrm{u}(\mathrm{t})-\mathrm{r}(\mathrm{t})^{*} \mathrm{u}(\mathrm{t}) \wedge 2-3^{*} \mathrm{p}(\mathrm{t})-\mathrm{r}(\mathrm{t})^{*} \mathrm{u}(\mathrm{t})^{\wedge} 4-8^{*} \mathrm{p}(\mathrm{t})^{*} \mathrm{u}(\mathrm{t}) \wedge 2-5^{*} \mathrm{p}(\mathrm{t})^{\wedge} 2 / \mathrm{r}(\mathrm{t}), \mathrm{t}\right) / \mathrm{u}$ $\left.\left.(\mathrm{t})^{\wedge} 2, \mathrm{t}\right)+\operatorname{diff}\left(\operatorname{diff}(\mathrm{v}(\mathrm{t}), \mathrm{t})^{*}\left(3^{*} \mathrm{r}(\mathrm{t})\right)^{*} \mathrm{u}(\mathrm{t}) \wedge 2+5^{*} \mathrm{p}(\mathrm{t})\right) / \mathrm{u}(\mathrm{t}) \wedge 2, \mathrm{t}\right)-2^{*} \mathrm{r}(\mathrm{t})^{*} \operatorname{diff}(\mathrm{v}(\mathrm{t}), \mathrm{t})^{*}$ $\mathrm{u}(\mathrm{t})-2^{*} \operatorname{diff}(\mathrm{r}(\mathrm{t}) * \operatorname{diff}(\mathrm{v}(\mathrm{t}), \mathrm{t}) / \mathrm{u}(\mathrm{t}), \mathrm{t})+2^{*} \operatorname{diff}(\mathrm{v}(\mathrm{t}), \mathrm{t})^{*}(\mathrm{r}(\mathrm{t}) * \operatorname{diff}(\mathrm{v}(\mathrm{t}), \mathrm{t})+\operatorname{diff}(\mathrm{p}(\mathrm{t})+\mathrm{r}$ $\left.\left.(t)^{*} u(t)^{\wedge} 2-r(t)^{*} u(t), t\right)\right) / u(t)^{\wedge} 2=0$,

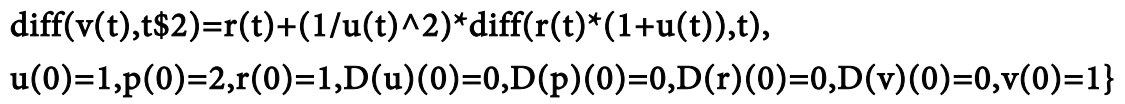

\section{Appendix 3}

The non-stationary wave attenuation of the spherical object without fields.

Let us consider the nonlocal space-time evolution of the spherical object. We suppose:

1) The nonlocal parameter $\tau=$ const .

2) Radial velocity of the object $v_{0 r}=$ const .

3) The thermal velocity $v_{T}=$ const, then

$$
p=\rho v_{T}^{2} .
$$

The nonlocal system of hydrodynamic equation has the following form-the continuity equation is [5]

$$
\frac{\partial^{2} \rho}{\partial t^{2}}+\frac{\partial^{2} p}{\partial r^{2}}+\frac{2}{r} \frac{\partial p}{\partial r}=\frac{1}{\tau} \frac{\partial \rho}{\partial t}
$$

and

$$
\frac{\partial^{2} p}{\partial t^{2}}+\frac{5}{3} \frac{\partial^{2}}{\partial r^{2}}\left(\frac{p^{2}}{\rho}\right)+\frac{10}{3 r} \frac{\partial}{\partial r}\left(\frac{p^{2}}{\rho}\right)=\frac{1}{\tau} \frac{\partial p}{\partial t} .
$$

The system consists of the continuity equation and the energy equation ( $\rho$ -density, $p$-quantum pressure). The equation of motion in a spherical coordinate system is absent if the radial component of the velocity is absent in the case of radial symmetry. Using (A3.1) we find in the dimensionless form; continuity equation 


$$
\frac{\partial^{2} \tilde{\rho}}{\partial \tilde{t}^{2}}+\frac{\partial^{2} \tilde{\rho}}{\partial \tilde{r}^{2}}+\frac{2}{\tilde{r}} \frac{\partial \tilde{\rho}}{\partial \tilde{r}}=\frac{\partial \tilde{\rho}}{\partial \tilde{t}}
$$

and

$$
\frac{\partial^{2} \tilde{p}}{\partial \tilde{t}^{2}}+\frac{\partial^{2} \tilde{p}}{\partial \tilde{r}^{2}}+\frac{2}{\tilde{r}} \frac{\partial \tilde{p}}{\partial \tilde{r}}=\frac{3}{5} \frac{\partial \tilde{p}}{\partial \tilde{t}} .
$$

The following scales are used: $t \leftrightarrow \tau, r \leftrightarrow v_{T} \tau$. Nonlinear parabolic Equations (A3.4), (A3.5) can be solved by Fourier method. Really, for (A3.4) we separate the unknown variables using

$$
\tilde{\rho}=R(\tilde{r}) T(\tilde{t}) .
$$

We find

$$
R(\tilde{r}) \frac{\partial T(\tilde{t})}{\partial \tilde{t}}=R(\tilde{r}) \frac{\partial^{2} T(\tilde{t})}{\partial \tilde{t}^{2}}+T(\tilde{t}) \frac{\partial^{2} R(\tilde{r})}{\partial \tilde{r}^{2}}+2 \frac{T(\tilde{t})}{\tilde{r}} \frac{\partial R(\tilde{r})}{\partial \tilde{r}}
$$

or

$$
\frac{1}{T(\tilde{t})} \frac{\partial T(\tilde{t})}{\partial \tilde{t}}-\frac{1}{T(\tilde{t})} \frac{\partial^{2} T(\tilde{t})}{\partial \tilde{t}^{2}}=\frac{1}{R(\tilde{r})} \frac{\partial^{2} R(\tilde{r})}{\partial \tilde{r}^{2}}+2 \frac{1}{\tilde{r} R(\tilde{r})} \frac{\partial R(\tilde{r})}{\partial \tilde{r}}
$$

Relations (A3.7) and (A3.8) lead to two ordinary differential equations

$$
\frac{\partial^{2} T(\tilde{t})}{\partial \tilde{t}^{2}}-\frac{\partial T(\tilde{t})}{\partial \tilde{t}}+C T(\tilde{t})=0
$$

and

$$
\frac{\partial^{2} R(\tilde{r})}{\partial \tilde{r}^{2}}+\frac{2}{\tilde{r}} \frac{\partial R(\tilde{r})}{\partial \tilde{r}}-C R(\tilde{r})=0
$$

If $C<0$

$$
T=\exp \left(\frac{1-\sqrt{1-4 C}}{2} \tilde{t}\right), C<0 .
$$

Equation (A3.10) can be solved by numerical methods. For example

$$
\tilde{r} \frac{\partial^{2} R(\tilde{r})}{\partial \tilde{r}^{2}}+2 \frac{\partial R(\tilde{r})}{\partial \tilde{r}}-C \tilde{r} R(\tilde{r})=0 .
$$

The solution for the quantum pressure can be found by the analogical way. It should be noticed that the solution of the homogeneous Equations (A3.4) and (A3.5) for unknown variables $p$ and $\rho$ can be found up to an additive arbitrary constant (Figure 14). 


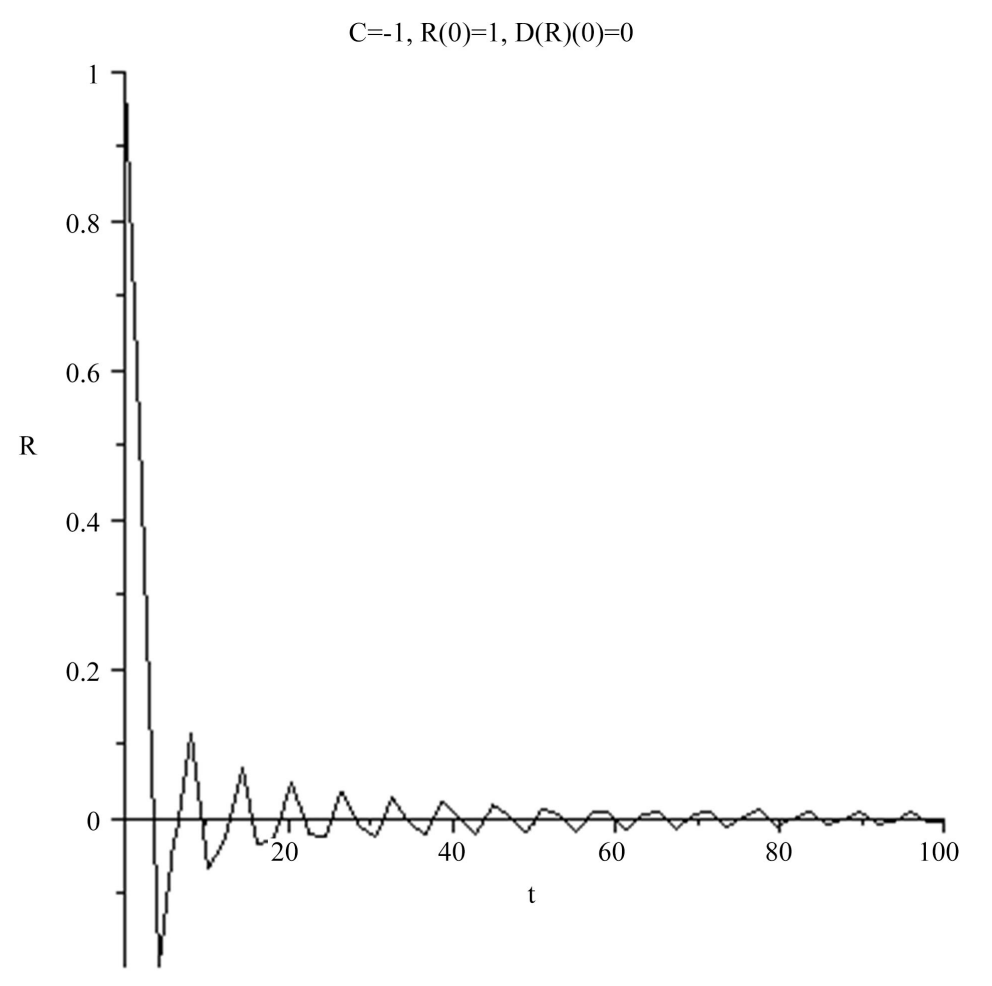

Figure 14. Radial density damping. 